\title{
RICCATI-BASED FEEDBACK CONTROL OF THE MONODOMAIN EQUATIONS WITH THE FITZHUGH-NAGUMO MODEL
}

\author{
TOBIAS BREITEN * AND KARL KUNISCH ${ }^{\dagger}$
}

\begin{abstract}
Feedback control for the monodomain equations is studied. The dynamics of interest are governed by a coupled PDE-ODE reaction diffusion system with non-monotone nonlinearity of FitzHugh-Nagumo type. A localized distributed control is used to locally stabilize the nonlinear system. This is achieved by a Riccati-based feedback law, determined by the linearized system. It is shown that the Riccati equation corresponding to the PDE variable suffices for exponential stabilization of the linearized PDE-ODE system. The theoretical findings are underlined by several numerical examples.
\end{abstract}

Key words. feedback control, Riccati equation, monodomain equations, FitzHugh-Nagumo model

AMS subject classifications. 35K57, 93B52, 93C20, 93D15

1. Introduction. Optimal control of reaction-diffusion type partial differential equations (PDEs) arising in cardiac electrophysiology has received considerable interest within the last few years, see, e.g., [9, 10, 12, 16, 17, 19]. The so-called bidomain equations are a well accepted model representing defibrillation processes of the human heart (see [33]). However, due to the complexity of the equations a reasonable alternative is to consider a simplification known as the monodomain equations ([25]). Here, the dynamics are governed by a parabolic reaction-diffusion system coupled with a linear ordinary differential equation modeling the ionic current. As has been discussed in, e.g., [17], under certain initial conditions, reentry phenomena and spiral waves can occur. From a medical point of view, these situations can be interpreted as fibrillation processes of the heart that should be terminated by an external control, e.g., by applying an external stimulus to the heart tissue (see [23]). With this in mind, the goal of this paper is to discuss the applicability of linear feedback laws for the following equations:

$$
\begin{aligned}
\frac{\partial v}{\partial t} & =\Delta v-I_{\text {ion }}(v, w)+f_{v}+B u, \quad \text { in } \Omega \times(0, \infty), \\
\frac{\partial w}{\partial t} & =\gamma v-\delta w+f_{w}, \quad \text { in } \Omega \times(0, \infty), \\
\frac{\partial v}{\partial \nu} & =0, \quad \text { on } \Gamma \times(0, \infty), \\
v(x, 0) & =v_{0}(x) \text { and } w(x, 0)=w_{0}(x), \text { in } \Omega,
\end{aligned}
$$

where $\Omega \subset \mathbb{R}^{n}, n \in\{2,3\}$, denotes a bounded open set with smooth boundary $\Gamma=\partial \Omega$. Here, $v=v(x, t)$ describes the transmembrane electrical potential, $w=w(x, t)$ is a socalled gating variable and $u=u(x, t) \in L^{2}\left(0, \infty ; L^{2}(\omega)\right)$, where $\omega \subset \Omega$, is a nonempty open subset, denotes the control. We further assume that $\gamma, \delta>0$ and denote by $\nu$ the outer unit normal to $\Gamma$. For fixed $t$, we define the linear operator $B \in \mathcal{L}\left(L^{2}(\omega), L^{2}(\Omega)\right)$

\footnotetext{
*Institute for Mathematics and Scientic Computing, Karl-Franzens-Universität, Heinrichstr. 36, 8010 Graz, Austria (tobias.breiten@uni-graz.at)

${ }^{\dagger}$ Institute for Mathematics and Scientic Computing, Karl-Franzens-Universität, Heinrichstr. 36, 8010 Graz, Austria (karl.kunisch@uni-graz.at)
} 


$$
(B u)(x)= \begin{cases}u(x), & x \in \omega, \\ 0, & x \in \Omega \backslash \omega .\end{cases}
$$

Its adjoint $B^{*}$ is the restriction operator from $\Omega$ to $\omega$. With slight abuse of notation, we also use $B$ as operator from $L^{2}\left(0, \infty ; L^{2}(\omega)\right) \rightarrow L^{2}\left(0, \infty ; L^{2}(\Omega)\right)$ with $(B u)(t)=$ $(B u(t))$ and analogously for $B^{*}$. In the above setting, the model for the ionic current $I_{\text {ion }}$ is given by a cubic nonlinearity and $f$ is assumed to be an external force.

Our goal now is the following. Given a stationary solution

$$
\begin{aligned}
& 0=\Delta \bar{v}-I_{\text {ion }}(\bar{v}, \bar{w})+f_{v}(x), \\
& 0=\gamma \bar{v}-\delta \bar{w}+f_{w}(x),
\end{aligned}
$$

we want to specify a linear feedback law of the form $u=k(v, w)$ such that $\left(y_{v}, y_{w}\right):=$ $(v-\bar{v}, w-\bar{w})$ is exponentially stable. In this paper, we follow a Riccati-based approach that recently has been studied for, e.g., the semilinear heat equation (see [3, 4]), the Navier-Stokes equations ([1, 2, 30,31]), the Burgers equation (see [32]) or the Boussinesq system $([8,15,24]$. While the open loop optimal control of reactiondiffusion type systems of the form (1.1) has been studied in $[6,9,18,19]$, less is known for feedback-based controls. In fact, by the authors knowledge, there do not exist any results on (local) feedback stabilizability of (1.1). In [6], an approximate controllability problem is discussed but the exact null controllability still seems to be an open question. On the other hand, for semilinear parabolic equations that are not coupled to a gating variable, controllability results are well-known, see, e.g., $[3,4]$. Based on these results, we propose to construct a feedback law by solving the algebraic Riccati equation associated with the decoupled system that is obtained for $w(x, t) \equiv 0$.

The structure of the paper now is as follows. In Section 2, we briefly recapitulate well-posedness as well as existence results for the monodomain equations (1.1). In Section 3, we derive the linearized system and show that the solution of the Riccati equation of the decoupled system suffices for exponential stabilization of the coupled system. We prove the local exponential stabilization of the full nonlinear system in Section 4 and underline the suggested approach by means of some numerical examples in Section 5.

In the remainder of this paper, we use the following notation. For $p \geq 1$ and $s \geq 0$, by $L^{p}(\Omega)$ and $H^{s}(\Omega)$ we denote the usual Lebesgue and Sobolev spaces. Additionally, we define $H_{\nu}^{s}(\Omega):=\left\{y \in \mathcal{D}(\Omega) \mid \frac{\partial y}{\partial \nu}=0\right.$ on $\left.\Gamma\right\}$, where the closure is taken with respect to $\|y\|_{H^{s}(\Omega)}, s \geq 0$. For $s>\frac{3}{2}$, we have $H_{\nu}^{s}=\left\{y \in H^{s}(\Omega) \mid \frac{\partial y}{\partial \nu}=0\right.$ on $\left.\Gamma\right\}$ and for $s \in$ $\left[0, \frac{3}{2}\right)$, we have $H_{\nu}^{s}(\Omega)=H^{s}(\Omega)$. Given a Hilbert space $X$, we denote with $L^{2}(0, \infty ; X)$ (Bochner) square integrable functions on $(0, \infty)$ with values in $X$. For $Q_{\infty}=\Omega \times(0, \infty)$ and $r \geq 0, s \geq 0$, we will need the spaces

$$
H^{r, s}\left(Q_{\infty}\right)=L^{2}\left(0, \infty ; H^{r}(\Omega)\right) \cap H^{s}\left(0, \infty ; L^{2}(\Omega)\right),
$$

which are Hilbert spaces with the norm

$$
\|u\|_{r, s}=\left(\int_{0}^{\infty}\|u(t)\|_{H^{r}(\Omega)}^{2} \mathrm{~d} t+\|u\|_{H^{s}\left(0, \infty ; L^{2}(\Omega)\right)}^{2}\right)^{\frac{1}{2}} .
$$


The space of bounded linear operators between two Hilbert spaces $X$ and $Y$ will be denoted by $\mathcal{L}(X, Y)$. For a closed densely defined operator $\mathcal{A}: \mathcal{D}(\mathcal{A}) \subset X \rightarrow X$, the resolvent set and the spectrum of $\mathcal{A}$ are denoted by $\rho(\mathcal{A})$ and $\sigma(\mathcal{A})$, respectively. The adjoint of $\mathcal{A}$ is denoted by $\mathcal{A}^{*}$.

2. Monodomain equations and the Schlögl model. Depending on the community and the considered model $I_{\text {ion }}(v, w)$ for the ionic current, equation (1.1) is referred to under different names such as the Schlögl or Nagumo system as well as the FitzHugh-Nagumo system. In this section, we briefly review known results concerning existence and uniqueness of solutions as well as optimal control problems related to (1.1).

The existence of solutions to the FitzHugh-Nagumo system have been studied for several years now. Early results are given in, e.g., [28, 29] where the authors discuss the existence as well as the qualitative behavior of solutions to the FitzHughNagumo equations. In [16], existence and regularity of solutions for irregular data are investigated.

In contrast to the previous works, [6] is one of the first references for optimal control problems related to the FitzHugh-Nagumo system. Based on the ionic model

$$
I_{\text {ion }}(v, w)=\left(v+\varphi_{1}(x, t)\right)\left(v+\varphi_{2}(x, t)\right)\left(v+\varphi_{3}(x, t)\right)-w,
$$

with functions $\varphi_{i}(x, t)$ being space and time dependent, the authors address quadratic optimal control problems. Using the Dubovitski-Milyoutin formalism, the optimality system is derived and associated controllability questions are discussed. In [7], the decoupled system, also known as the Schlögl equation, is studied and the optimal control problem is numerically solved by using a model reduction technique (POD). In [19], the authors focus on a time optimal control problem for a linearized version of the FitzHugh-Nagumo system, i.e., the ionic model is given by $I_{\text {ion }}(v, w)=\varphi(x) v+w$ and the control function is subject to pointwise control constraints. For general ionic models of the form

$$
I_{\text {ion }}(v, w)=k\left(v-\varphi_{1}\right)\left(v-\varphi_{2}\right)\left(v-\varphi_{3}\right)+\alpha w,
$$

in [9], the authors discuss the effect of controls acting only in a small part of the domain $\Omega$. Due to a resulting $L^{1}$ norm term, here the corresponding sparse optimal control problem yields a non-differentiable objective function. Finally, we mention [18], where a modified version of the FitzHugh-Nagumo model, known as the RogersMcCulloch model is studied. In that case, the ionic model is given as

$$
I_{\text {ion }}(v, w)=\frac{\eta_{0}}{v_{t h} v_{p k}} v^{3}-\eta_{0}\left(\frac{1}{v_{t h}}+\frac{1}{v_{p k}}\right) v^{2}+\eta_{0} v+\eta_{1} v w,
$$

where the parameters $\eta_{0}, \eta_{1}, \eta_{2}>0$ are assumed to be real constants and $v_{p k}>v_{t h}>0$ denote peak and threshold potential. Further numerical studies and error estimates are discussed in, e.g., $[10,12,17]$. In this paper, we consider the FitzHugh-Nagumo model in the form

$$
I_{\text {ion }}(v, w)=a v^{3}+b v^{2}+c v+d w
$$

where $a, b, c, d \in \mathbb{R}$ are constant parameters. We point out that none of the above mentioned articles deals with feedback based control functions $u(x, t)=k(v(x, t), w(x, t))$. In particular, control theoretic approaches given by the solution of Riccati equations 
have not been investigated up to now. A difficulty for the latter method clearly is the lack of controllability and stabilizability results for reaction-diffusion type systems of the form (1.1). While [6] gives an approximate controllability result, the question of stabilizability for the FitzHugh-Nagumo system has not been answered yet. In the subsequent sections, we follow the methodology from [31], where the local exponential stabilization of the Burgers equation is addressed.

3. Stabilizability of the linearized system. Since we want to apply a Riccatibased feedback control law, we are going to rewrite the system as an abstract Cauchy problem of the form

$$
\vec{y}^{\prime}(t)=\mathcal{A} \vec{y}(t)+\mathcal{F}(\vec{y}(t))+\mathcal{B} u(t)
$$

where $\mathcal{A}$ is the infinitesimal generator of a strongly continuous semigroup on a Hilbert space $\mathcal{Y}, \mathcal{F}$ is a nonlinear operator and $\mathcal{B}: \mathcal{U} \rightarrow \mathcal{Y}$ is the control operator mapping from the control space $\mathcal{U}$ to the state space $\mathcal{Y}$. Our goal is to apply a control of the form $u(t)=-\mathcal{B}^{*} \Pi \vec{y}(t)$ with $\Pi=\Pi^{*} \succeq 0$ denoting the solution to the algebraic Riccati equation

$$
\mathcal{A}^{*} \Pi+\Pi \mathcal{A}-\Pi \mathcal{B} \mathcal{B}^{*} \Pi+\mathcal{I}=0 .
$$

In what follows, for the stationary solution to $(1.2)$, we assume that $(\bar{v}, \bar{w}) \in H^{\frac{n}{2}+s}(\Omega) \times$ $L^{\infty}(\Omega), s>0$. In order to obtain the structure in (3.1), we define the difference $\left(y_{v}, y_{w}\right):=(v-\bar{v}, w-\bar{w})$ and obtain

$$
\begin{aligned}
\frac{\partial y_{v}}{\partial t} & =\Delta y_{v}-a\left(v^{3}-\bar{v}^{3}\right)-b\left(v^{2}-\bar{v}^{2}\right)-c y_{v}-d y_{w}+B u \\
\frac{\partial y_{w}}{\partial t} & =\gamma y_{v}-\delta y_{w}
\end{aligned}
$$

Using the identities

$$
\begin{aligned}
& v^{3}-\bar{v}^{3}=\left(y_{v}+\bar{v}\right)^{3}-\bar{v}^{3}=y_{v}^{3}+3 \bar{v} y_{v}^{2}+3 \bar{v}^{2} y_{v}, \\
& v^{2}-\bar{v}^{2}=\left(y_{v}+\bar{v}\right)^{2}-\bar{v}^{2}=y_{v}^{2}+2 \bar{v} y_{v},
\end{aligned}
$$

we can cast (3.3) into the form of (3.1). For this, we denote $\vec{y}:=\left(y_{v}, y_{w}\right)$ and the operators

$$
\begin{gathered}
\mathcal{A} \vec{y}=\left(\begin{array}{c}
\Delta y_{v}-\left(3 a \bar{v}^{2}+2 b \bar{v}+c\right) y_{v}-d y_{w} \\
\gamma y_{v}-\delta y_{w}
\end{array}\right), \\
\mathcal{F}(\vec{y})=\left(\begin{array}{c}
-a y_{v}^{3}-(b+3 a \bar{v}) y_{v}^{2} \\
0
\end{array}\right), \quad \mathcal{B} u=\left(\begin{array}{c}
B u \\
0
\end{array}\right) .
\end{gathered}
$$

Our first result now shows that the operator $\mathcal{A}$ generates an analytic semigroup on $L^{2}(\Omega) \times L^{2}(\Omega)$. This is essential for the rest of the paper since we can make use of some beneficial properties of analytic semigroups that we mention later on.

Lemma 3.1. The operator

$$
\begin{aligned}
\mathcal{A} & : \mathcal{D}(\mathcal{A}) \subset L^{2}(\Omega) \times L^{2}(\Omega) \rightarrow L^{2}(\Omega) \times L^{2}(\Omega), \\
\mathcal{D}(\mathcal{A}) & =\left\{\vec{y} \in H^{2}(\Omega) \times L^{2}(\Omega) \mid \frac{\partial y_{v}}{\partial \nu}=0 \text { on } \Gamma\right\}
\end{aligned}
$$


is the infinitesimal generator of an analytic semigroup on $L^{2}(\Omega) \times L^{2}(\Omega)$.

Proof. The proof follows the lines of the proof [19, Lemma 2.2]. Assume that $\lambda_{0}>0$. First, from [5, Theorem 2.12], we know that the operator

$$
\Delta-\lambda_{0} I, \quad \mathcal{D}\left(\Delta-\lambda_{0} I\right)=\left\{y \in H^{2}(\Omega) \mid \frac{\partial y}{\partial \nu}=0\right\}
$$

generates an analytic semigroup $S(t)$ on $L^{2}(\Omega)$. Also, it holds that $S(t)$ is differentiable and, since $0 \in \rho\left(\Delta-\lambda_{0} I\right)$, using [26, Theorem 6.13, Chapter 2] we conclude the existence of a constant $C$ such that for all $t>0$ and $y \in L^{2}(\Omega)$, we have

$$
\left\|\left(\Delta-\lambda_{0} I\right) S(t) y\right\|_{L^{2}(\Omega)} \leq \frac{C}{t}\|y\|_{L^{2}(\Omega)} .
$$

Following [19], for $t \geq 0$, we define the operator $\tilde{S}(t)$ from $L^{2}(\Omega) \times L^{2}(\Omega)$ via $\tilde{S}(t) \vec{y}=$ $\left(\begin{array}{c}S(t) y_{v} \\ 0\end{array}\right)$ and note that $\tilde{S}(t)$ is a $C_{0}$ semigroup on $L^{2}(\Omega) \times L^{2}(\Omega)$ with infinitesimal generator

$$
\mathcal{A}_{0} \vec{y}=\left(\begin{array}{c}
\Delta y_{v}-\lambda_{0} y_{v} \\
0
\end{array}\right), \quad \mathcal{D}\left(\mathcal{A}_{0}\right)=\mathcal{D}\left(\Delta-\lambda_{0} I\right) \times L^{2}(\Omega) .
$$

Using the previous estimate for $S(t)$, we also have that

$$
\left\|\mathcal{A}_{0} \tilde{S}(t) \vec{y}\right\|_{L^{2}(\Omega) \times L^{2}(\Omega)} \leq \frac{C}{t}\|\vec{y}\|_{L^{2}(\Omega) \times L^{2}(\Omega)} .
$$

According to [26, Theorem 5.2, Chapter 2] this implies that $\tilde{S}(t)$ is an analytic semigroup on $L^{2}(\Omega) \times L^{2}(\Omega)$. Now, we use the splitting $\mathcal{A}=\mathcal{A}_{0}+\mathcal{A}_{1}$, where

$$
\mathcal{A} \vec{y}=\mathcal{A}_{0} \vec{y}+\mathcal{A}_{1} \vec{y}=\left(\begin{array}{c}
\Delta y_{v}-\lambda_{0} y_{v} \\
0
\end{array}\right)+\left(\begin{array}{c}
\lambda_{0} y_{v}-\left(3 a \bar{v}^{2}+2 b \bar{v}+c\right) y_{v}-d y_{w} \\
\gamma y_{v}-\delta y_{w}
\end{array}\right)
$$

and $\mathcal{D}\left(\mathcal{A}_{1}\right)=L^{2}(\Omega) \times L^{2}(\Omega)$. Since $\bar{v} \in L^{\infty}(\Omega)$, the operator $\mathcal{A}_{1}$ is bounded from $L^{2}(\Omega) \times L^{2}(\Omega)$ to itself and the assertion follows from [26].

As we already mentioned, the analyticity of the semigroup is essential for our purposes since we can make use of the spectrum determined growth assumption ([26, Corollary 2.2]). This means that $\sup _{\lambda \in \sigma(\mathcal{A})} \lambda$ coincides with the growth bound of the generated semigroup. The question of stabilizability for the linearized system associated with $\mathcal{A}$, however, is still not answered. We address this problem by using existing results for the decoupled system

$$
\begin{aligned}
& \frac{\partial y_{v}}{\partial t}=\Delta y_{v}-\left(3 a \bar{v}^{2}+2 b \bar{v}+c\right) y_{v}+B u, \text { in } \Omega \times(0, \infty), \\
& \frac{\partial y_{v}}{\partial \nu}=0, \quad \text { on } \Gamma \times(0, \infty) .
\end{aligned}
$$

From [11], we know that the system is null controllable with localized distributed controls. Hence, if we define

$$
\begin{gathered}
A_{v}: \mathcal{D}\left(A_{v}\right) \subset L^{2}(\Omega) \rightarrow L^{2}(\Omega), \\
\mathcal{D}\left(A_{v}\right)=\left\{y \in H^{2}(\Omega) \mid \frac{\partial y_{v}}{\partial \nu}=0 \text { on } \Gamma\right\}
\end{gathered}
$$


via

$$
A_{v} y_{v}=\Delta y_{v}-\left(3 a \bar{v}^{2}+2 b \bar{v}+c\right) y_{v},
$$

from $[27,34]$ we know that $(3.5)$ is exponentially stabilizable. Since the results from [11] apply also to the shifted operator $A_{v}+\alpha I$ for every $\alpha>0$, with, e.g., [34], it follows that there exists a unique positive solution $\Pi=\Pi^{*} \in \mathcal{L}\left(L^{2}(\Omega)\right)$ to

$$
\left(A_{v}+\alpha I\right)^{*} \Pi+\Pi\left(A_{v}+\alpha I\right)-\Pi B B^{*} \Pi+I=0 .
$$

Also, the semigroup generated by $A_{v}+\alpha I-B B^{*} \Pi$ is exponentially stable, i.e., its growth bound is negative. Equivalently, this implies that the semigroup generated by $A_{v}-B B^{*} \Pi$ has a growth bound that is smaller than $-\alpha$. We now use the exponential stabilizability of the decoupled system for the stabilization of the coupled system. We have the following result.

Lemma 3.2. Let $\Pi$ be the solution to (3.7) with $\alpha=\varepsilon+\gamma \frac{|d|}{\delta-\varepsilon}, 0<\varepsilon<\delta$. Define the operator $\left(\mathcal{A}_{\Pi}, \mathcal{D}\left(\mathcal{A}_{\Pi}\right)\right)$ as follows

$$
\begin{aligned}
\mathcal{A}_{\Pi} \vec{y} & =\left(\begin{array}{c}
A_{v} y_{v}-d y_{w}-B B^{*} \Pi y_{v} \\
\gamma y_{v}-\delta y_{w}
\end{array}\right), \\
\mathcal{D}\left(\mathcal{A}_{\Pi}\right) & =\left\{\vec{y} \in L^{2}(\Omega) \times L^{2}(\Omega) \mid y_{v} \in \mathcal{D}\left(A_{v}\right)\right\} \\
& =\left\{\vec{y} \in H^{2}(\Omega) \times L^{2}(\Omega) \mid \frac{\partial y_{v}}{\partial \nu}=0 \text { on } \Gamma\right\} .
\end{aligned}
$$

Then $\mathcal{A}_{\Pi}$ is the infinitesimal generator of an exponentially stable analytic semigroup on $L^{2}(\Omega) \times L^{2}(\Omega)$. In particular, for its growth bound $\omega$ it holds that $\omega \leq-\varepsilon$.

Proof. From [5, Proposition 2.3] we know that for $\lambda \in \mathbb{C}$ with $\operatorname{Re}(\lambda) \geq-\varepsilon-\gamma \frac{|d|}{\delta-\varepsilon}$ the resolvent $\left(\lambda I-\left(A_{v}-B B^{*} \Pi_{v}\right)\right)^{-1}$ exists. Assume now that $s \in \mathbb{C}$ with $\operatorname{Re}(s) \geq-\varepsilon$ and define $z:=s+\frac{d}{s+\delta} \gamma$. The real part of $z$ then is given as

$$
\operatorname{Re}(z)=\operatorname{Re}(s)+\frac{d \gamma(\operatorname{Re}(s)+\delta)}{(\operatorname{Re}(s)+\delta)^{2}+\operatorname{Im}(s)^{2}} .
$$

Since $\operatorname{Re}(s) \geq-\varepsilon$, this implies

$$
\operatorname{Re}(z) \geq-\varepsilon-\frac{|d| \gamma}{\operatorname{Re}(s)+\delta} \geq-\varepsilon-\gamma \frac{|d|}{\delta-\varepsilon},
$$

and we conclude that $\left(z I-\left(A_{v}-B B^{*} \Pi\right)\right)^{-1}=\left(s I+\frac{d}{s+\delta} \gamma I-\left(A_{v}-B B^{*} \Pi\right)\right)^{-1}$ exists for $\operatorname{Re}(s) \geq-\varepsilon$. Next, let a partitioning be given as follows

$$
\left(s I-\mathcal{A}_{\Pi}\right):=\left(\begin{array}{ll}
\tilde{A} & \tilde{B} \\
\tilde{C} & \tilde{D}
\end{array}\right)=\left(\begin{array}{cc}
s I-A_{v}+B B^{*} \Pi & d I \\
-\gamma I & (s+\delta) I
\end{array}\right) .
$$

According to the preceding arguments and the fact that $\delta>\varepsilon$, we know that $\tilde{A}^{-1}, \tilde{D}^{-1}$ and the Schur complement $\left(\tilde{A}-\tilde{B} \tilde{D}^{-1} \tilde{C}\right)^{-1}$ exist for $\operatorname{Re}(s) \geq-\varepsilon$. This particularly shows the existence of $\left(s I-\mathcal{A}_{\Pi}\right)^{-1}$ for $\operatorname{Re}(s) \geq-\varepsilon$. Since $\mathcal{A}$ is the infinitesimal generator of an analytic semigroup and $B B^{*} \Pi$ is bounded, $\mathcal{A}_{\Pi}$ also generates an analytic semigroup. This means the spectrum determined growth assumption holds and we can conclude that the semigroup generated by $\mathcal{A}_{\Pi}$ is exponentially stable with growth bound $\omega \leq-\varepsilon$. 


\section{Local stabilization of the nonlinear system.}

4.1. Properties of the nonlinear function $\mathcal{F}$. In order to show the local stabilization of the nonlinear system (3.1), we first need some properties of the nonlinear operator $\mathcal{F}$. Following the approach in [30, 31], we investigate local Lipschitz continuity. With this in mind, we start with a result concerning the norm of the space $H^{r, s}\left(Q_{\infty}\right)$. For the remainder of this paper, $C$ denotes a generic constant that might change its value depending on the specific estimate.

LEMMA 4.1. Let $f, g, h \in H^{\lambda+2 \mu, \frac{\lambda+2 \mu}{2}}\left(Q_{\infty}\right)$. If

(i) $\lambda+3 \mu \geq \frac{n+2}{2}$,

(ii) $2 \lambda+4 \mu \geq 0, \mu \geq 0$,

and $(i)$ is strict if equality holds in one of the inequalities in (ii), then

$$
\begin{aligned}
\|f \cdot g\|_{\lambda, \frac{\lambda}{2}} & \leq C \cdot\|f\|_{\lambda+2 \mu, \frac{\lambda+2 \mu}{2}} \cdot\|g\|_{\lambda+2 \mu, \frac{\lambda+2 \mu}{2}}, \\
\|f \cdot g \cdot h\|_{\lambda, \frac{\lambda}{2}} & \leq C \cdot\|f\|_{\lambda+2 \mu, \frac{\lambda+2 \mu}{2}} \cdot\|g\|_{\lambda+2 \mu, \frac{\lambda+2 \mu}{2}} \cdot\|h\|_{\lambda+2 \mu, \frac{\lambda+2 \mu}{2}} .
\end{aligned}
$$

Note that for $\lambda, \mu \geq 0$, the definition of $H^{\lambda, \mu}\left(Q_{\infty}\right)$ from [14] coincides with our definition of $H^{r, s}\left(Q_{\infty}\right)$.

Proof. We know that $\lambda+2 \mu+2 \mu \geq \frac{n+2}{2}$. Moreover, since $2 \mu \geq 0$ and $2 \lambda+2 \mu+2 \mu \geq$ 0 , the first assertions follows from [14, Theorem B.3]. Analogously, it holds that

$$
\|g \cdot h\|_{\lambda+\mu, \frac{\lambda+\mu}{2}} \leq C \cdot\|g\|_{\lambda+2 \mu, \frac{\lambda+2 \mu}{2}} \cdot\|h\|_{\lambda+2 \mu, \frac{\lambda+2 \mu}{2}}
$$

since for $\mu \geq 0$, we have $(\lambda+\mu)+\mu+\mu \geq \frac{n+2}{2}$ and $2(\lambda+\mu)+\mu+\mu \geq 0$. Finally, due to (i) and (ii), again with [14] we get

$$
\|f \cdot g \cdot h\|_{\lambda, \frac{\lambda}{2}} \leq C \cdot\|f\|_{\lambda+2 \mu, \frac{\lambda+2 \mu}{2}} \cdot\|g \cdot h\|_{\lambda+\mu, \frac{\lambda+\mu}{2}}
$$

which, combined with (4.1), shows the second assertion.

First we consider the two-dimensional case and give a useful estimate for the difference of quadratic and cubic terms as they appear for $\mathcal{F}$.

Lemma 4.2. Let $\Omega \subset \mathbb{R}^{2}$ and assume that $\varepsilon \in\left[\frac{1}{3}, 1\right]$. Then for $y_{v}, z_{v} \in H^{1+\varepsilon, \frac{1+\varepsilon}{2}}\left(Q_{\infty}\right)$, it holds that

$$
\begin{aligned}
& \left\|y_{v}^{2}-z_{v}^{2}\right\|_{L^{2}\left(0, \infty ; H^{\varepsilon-1}(\Omega)\right)} \leq C \cdot\left\|y_{v}-z_{v}\right\|_{1+\varepsilon, \frac{1+\varepsilon}{2}}\left(\left\|y_{v}\right\|_{1+\varepsilon, \frac{1+\varepsilon}{2}}+\left\|z_{v}\right\|_{1+\varepsilon, \frac{1+\varepsilon}{2}}\right), \\
& \left\|y_{v}^{3}-z_{v}^{3}\right\|_{L^{2}\left(0, \infty ; H^{\varepsilon-1}(\Omega)\right)} \leq C \cdot\left\|y_{v}-z_{v}\right\|_{1+\varepsilon, \frac{1+\varepsilon}{2}}\left(\left\|y_{v}\right\|_{1+\varepsilon, \frac{1+\varepsilon}{2}}^{2}+\left\|z_{v}\right\|_{1+\varepsilon, \frac{1+\varepsilon}{2}}^{2}\right) .
\end{aligned}
$$

Proof. For $\varepsilon \in\left[\frac{1}{3}, 1\right)$, we choose $\lambda=3 \varepsilon-1, \mu=1-\varepsilon$. Then, $\mu \in\left(0, \frac{2}{3}\right], \lambda+2 \mu=$ $1+\varepsilon>0$ and $\lambda+3 \mu=2$ and Lemma 4.1 implies

$$
\begin{aligned}
\left\|y_{v}^{2}-z_{v}^{2}\right\|_{3 \varepsilon-1, \frac{3 \varepsilon-1}{2}} & =\left\|\left(y_{v}-z_{v}\right)\left(y_{v}+z_{v}\right)\right\|_{3 \varepsilon-1, \frac{3 \varepsilon-1}{2}} \\
& \leq C\left\|y_{v}-z_{v}\right\|_{1+\varepsilon, \frac{1+\varepsilon}{2}}\left(\left\|y_{v}\right\|_{1+\varepsilon, \frac{1+\varepsilon}{2}}+\left\|z_{v}\right\|_{1+\varepsilon, \frac{1+\varepsilon}{2}}\right) .
\end{aligned}
$$

Analogously, due to Lemma 4.1 and Young's inequality, it holds

$$
\begin{aligned}
\left\|y_{v}^{3}-z_{v}^{3}\right\|_{3 \varepsilon-1, \frac{3 \varepsilon-1}{2}} & =\left\|\left(y_{v}-z_{v}\right)\left(y_{v}^{2}+y_{v} z_{v}+z_{v}^{2}\right)\right\|_{3 \varepsilon-1, \frac{3 \varepsilon-1}{2}} \\
& \leq C\left\|y_{v}-z_{v}\right\|_{1+\varepsilon, \frac{1+\varepsilon}{2}}\left(\left\|y_{v}\right\|_{1+\varepsilon, \frac{1+\varepsilon}{2}}^{2}+\left\|z_{v}\right\|_{1+\varepsilon, \frac{1+\varepsilon}{2}}^{2} .\right.
\end{aligned}
$$


Finally, boundedness of $\Omega$ implies $H^{3 \varepsilon-1}(\Omega) \subset H^{\varepsilon-1}(\Omega)$ which shows the assertion for $\varepsilon<1$. For $\varepsilon=1$, we choose $\lambda=0$ and $\mu=1$. Then the above splitting together with Lemma 4.1 yields the assertion.

Additionally, for the three-dimensional setting we have a similar result.

Lemma 4.3. Let $\Omega \subset \mathbb{R}^{3}$. For $y_{v}, z_{v} \in H^{2,1}\left(Q_{\infty}\right)$, it holds

$$
\begin{aligned}
& \left\|y_{v}^{2}-z_{v}^{2}\right\|_{L^{2}\left(Q_{\infty}\right)} \leq C \cdot\left\|y_{v}-z_{v}\right\|_{2,1}\left(\left\|y_{v}\right\|_{2,1}+\left\|z_{v}\right\|_{2,1}\right), \\
& \left\|y_{v}^{3}-z_{v}^{3}\right\|_{L^{2}\left(Q_{\infty}\right)} \leq C \cdot\left\|y_{v}-z_{v}\right\|_{2,1}\left(\left\|y_{v}\right\|_{2,1}^{2}+\left\|z_{v}\right\|_{2,1}^{2}\right) .
\end{aligned}
$$

Proof. Due to the triangle inequality, we have

$$
\begin{aligned}
\left\|y_{v}^{2}-z_{v}^{2}\right\|_{L^{2}\left(Q_{\infty}\right)} & =\left\|\left(y_{v}-z_{v}\right)\left(y_{v}+z_{v}\right)\right\|_{L^{2}\left(Q_{\infty}\right)} \\
& \leq C\left(\left\|\left(y_{v}-z_{v}\right) y_{v}\right\|_{L^{2}\left(Q_{\infty}\right)}+\left\|\left(y_{v}-z_{v}\right) z_{v}\right\|_{L^{2}\left(Q_{\infty}\right)}\right)
\end{aligned}
$$

as well as

$$
\begin{aligned}
\left\|y_{v}^{3}-z_{v}^{3}\right\|_{L^{2}\left(Q_{\infty}\right)}= & \left\|\left(y_{v}-z_{v}\right)\left(y_{v}^{2}+y_{v} z_{v}+z_{v}^{2}\right)\right\|_{L^{2}\left(Q_{\infty}\right)} \\
\leq C & \left(\left\|\left(y_{v}-z_{v}\right) y_{v}^{2}\right\|_{L^{2}\left(Q_{\infty}\right)}\right. \\
& \left.\quad+\left\|\left(y_{v}-z_{v}\right) y_{v} z_{v}\right\|_{L^{2}\left(Q_{\infty}\right)}+\left\|\left(y_{v}-z_{v}\right) z_{v}^{2}\right\|_{L^{2}\left(Q_{\infty}\right)}\right)
\end{aligned}
$$

We now choose $\lambda=0$ and $\mu=1$. Then $\lambda+3 \mu>\frac{5}{2}$. Again Lemma 4.1 and Young's inequality applied to the second term implies the claim.

With the previous results, we can show that $\mathcal{F}$ is locally Lipschitz continuous.

Lemma 4.4. Let $\Omega \subset \mathbb{R}^{2}$. Assume that $\varepsilon \in\left[\frac{1}{3}, 1\right]$. If

$$
\vec{y}, \vec{z} \in H^{1+\varepsilon, \frac{1+\varepsilon}{2}}\left(Q_{\infty}\right) \times\left(C\left([0, \infty] ; H^{1+\varepsilon}(\Omega)\right) \cap H^{1}\left(0, \infty ; H^{1+\varepsilon}(\Omega)\right)\right),
$$

then $\mathcal{F}$ is locally Lipschitz continuous from

$$
H^{1+\varepsilon, \frac{1+\varepsilon}{2}}\left(Q_{\infty}\right) \times C\left([0, \infty] ; H^{1+\varepsilon}(\Omega)\right) \cap H^{1}\left(0, \infty ; H^{1+\varepsilon}(\Omega)\right)
$$

to $L^{2}\left(0, \infty ; H^{\varepsilon-1}(\Omega)\right) \times L^{2}\left(Q_{\infty}\right)$. More precisely, it holds that

$$
\begin{aligned}
& \|\mathcal{F}(\vec{y})-\mathcal{F}(\vec{z})\|_{L^{2}\left(0, \infty ; H^{\varepsilon-1}(\Omega)\right) \times L^{2}\left(Q_{\infty}\right)} \\
& \quad \leq C_{1} \cdot\left(\left\|y_{v}\right\|_{1+\varepsilon, \frac{1+\varepsilon}{2}}+\left\|z_{v}\right\|_{1+\varepsilon, \frac{1+\varepsilon}{2}}+\left\|y_{v}\right\|_{1+\varepsilon, \frac{1+\varepsilon}{2}}^{2}+\left\|z_{v}\right\|_{1+\varepsilon, \frac{1+\varepsilon}{2}}^{2}\right)\left\|y_{v}-z_{v}\right\|_{1+\varepsilon, \frac{1+\varepsilon}{2}}
\end{aligned}
$$

Proof. Due to the structure of $\mathcal{F}$, it holds

$$
\begin{aligned}
& \|\mathcal{F}(\vec{y})-\mathcal{F}(\vec{z})\|_{L^{2}\left(0, \infty ; H^{\varepsilon-1}(\Omega)\right) \times L^{2}\left(Q_{\infty}\right)} \\
& \quad=\left\|-(b+3 a \bar{v})\left(y_{v}^{2}-z_{v}^{2}\right)-a\left(y_{v}^{3}-z_{v}^{3}\right)\right\|_{L^{2}\left(0, \infty ; H^{\varepsilon-1}(\Omega)\right)} \\
& \quad \leq C_{1}\left\|(b+3 a \bar{v})\left(y_{v}^{2}-z_{v}^{2}\right)\right\|_{L^{2}\left(0, \infty ; H^{\varepsilon-1}(\Omega)\right)}+\left\|a\left(y_{v}^{3}-z_{v}^{3}\right)\right\|_{L^{2}\left(0, \infty ; H^{\varepsilon-1}(\Omega)\right)}
\end{aligned}
$$

Since $\bar{v} \in H^{1+s}(\Omega), s>0$, with [14, Proposition B.1], we further conclude that

$$
\leq C_{2}\left(\left\|y_{v}^{2}-z_{v}^{2}\right\|_{L^{2}\left(0, \infty ; H^{\varepsilon-1}(\Omega)\right)}+\left\|y_{v}^{3}-z_{v}^{3}\right\|_{L^{2}\left(0, \infty ; H^{\varepsilon-1}(\Omega)\right)}\right)
$$

Lemma 4.2 immediately shows the assertion. $\square$ 
For the sake of completeness, we state the result for the three-dimensional case. LeMma 4.5. Let $\Omega \subset \mathbb{R}^{3}$. If

$$
\vec{y}, \vec{z} \in H^{2,1}\left(Q_{\infty}\right) \times C\left([0, \infty] ; H^{2}(\Omega)\right) \cap H^{1}\left(0, \infty ; H^{2}(\Omega)\right),
$$

then $\mathcal{F}$ is locally Lipschitz continuous from

$$
H^{2,1}\left(Q_{\infty}\right) \times C\left([0, \infty] ; H^{2}(\Omega)\right) \cap H^{1}\left(0, \infty ; H^{2}(\Omega)\right)
$$

to $L^{2}\left(Q_{\infty}\right) \times L^{2}\left(Q_{\infty}\right)$. More precisely, it holds

$$
\begin{aligned}
& \|\mathcal{F}(\vec{y})-\mathcal{F}(\vec{z})\|_{L^{2}\left(Q_{\infty}\right) \times L^{2}\left(Q_{\infty}\right)} \\
& \quad \leq \tilde{C}_{1} \cdot\left(\left\|y_{v}\right\|_{2,1}+\left\|z_{v}\right\|_{2,1}+\left\|y_{v}\right\|_{2,1}^{2}+\left\|z_{v}\right\|_{2,1}^{2}\right)\left\|y_{v}-z_{v}\right\|_{2,1} .
\end{aligned}
$$

As we have seen, for both the two-dimensional and the three-dimensional case, $\mathcal{F}$ is a locally Lipschitz continuous function. Together with results below, this will guarantee the locally exponential stabilizability of the system.

4.2. Nonhomogeneous equations. Before we can show local stabilization of the nonlinear system, we need some regularity results for the nonhomogeneous equation

$$
\vec{y}^{\prime}=\mathcal{A}_{\Pi} \vec{y}+\vec{f}, \quad \vec{y}(0)=\vec{y}_{0} .
$$

The first main result of this section is Theorem 4.7 which extends [31, Theorem 4.1] to $\varepsilon \in\left(\frac{1}{2}, 1\right]$. Note that this is essential for our purposes since the three-dimensional case indeed requires $\varepsilon=1$. For the proof, we recall a result from [5, Chapter 3, Theorem $2.2]$, stated here for an infinite time interval.

THEOREM 4.6. Let $\mathcal{Y}$ be a Hilbert space and suppose that $\mathcal{A}$ is the infinitesimal generator of an analytic semigroup of negative type on $\mathcal{Y}$. Then, for all $0 \leq \alpha \leq 1$, the mapping

$$
\begin{aligned}
& y \mapsto\left(y^{\prime}-\mathcal{A} y, y(0)\right) \\
& \quad L^{2}\left(0, \infty ;[\mathcal{D}(\mathcal{A}), \mathcal{Y}]_{\alpha}\right) \cap H^{1}\left(0, \infty ;\left[\mathcal{D}\left(\mathcal{A}^{*}\right), \mathcal{Y}\right]_{1-\alpha}^{*}\right) \\
& \quad \rightarrow L^{2}\left(0, \infty ;\left[\mathcal{D}\left(\mathcal{A}^{*}\right), \mathcal{Y}\right]_{1-\alpha}^{*}\right) \times\left[[\mathcal{D}(\mathcal{A}), \mathcal{Y}]_{\alpha},\left[\mathcal{D}\left(\mathcal{A}^{*}\right), \mathcal{Y}\right]_{1-\alpha}^{*}\right]_{\frac{1}{2}}
\end{aligned}
$$

is an isomorphism.

For the solution to (4.2), we now get the following result.

THEOREM 4.7. Let $\varepsilon \in\left(\frac{1}{2}, 1\right]$. If $\vec{f} \in L^{2}\left(0, \infty ; H^{\varepsilon-1}(\Omega)\right) \times L^{2}\left(Q_{\infty}\right), \vec{y}_{0} \in H^{\varepsilon}(\Omega) \times$ $H^{1+\varepsilon}(\Omega)$, then (4.2) has a unique solution

$$
\vec{y} \in H^{1+\varepsilon, \frac{1+\varepsilon}{2}}\left(Q_{\infty}\right) \times\left(C\left([0, \infty] ; H^{1+\varepsilon}(\Omega)\right) \cap H^{1}\left(0, \infty ; H^{1+\varepsilon}(\Omega)\right)\right)
$$

satisfying

$\|\vec{y}\|_{H^{1+\varepsilon, \frac{1+\varepsilon}{2}}\left(Q_{\infty}\right) \times H^{1}\left(0, \infty ; H^{1+\varepsilon}(\Omega)\right)} \leq C_{2}\left(\left\|\vec{y}_{0}\right\|_{H^{\varepsilon}(\Omega) \times H^{1+\varepsilon}(\Omega)}+\|\vec{f}\|_{L^{2}\left(0, \infty ; H^{\varepsilon-1}(\Omega)\right) \times L^{2}\left(Q_{\infty}\right)}\right)$.

Proof. Step 1: First of all, since $(\bar{v}, \bar{w}) \in L^{\infty}(\Omega) \times L^{\infty}(\Omega)$, we can choose $\lambda_{0}>0$ such that

$$
\begin{gathered}
\left(\left(\lambda_{0} I-\mathcal{A}\right) \vec{y}, \vec{y}\right)_{L^{2}(\Omega) \times L^{2}(\Omega)} \geq 0, \quad \text { for all } \vec{y} \in \mathcal{D}(\mathcal{A}) \\
\left(\left(\lambda_{0} I-\mathcal{A}^{*}\right) \vec{y}, \vec{y}\right)_{L^{2}(\Omega) \times L^{2}(\Omega)} \geq 0, \quad \text { for all } \vec{y} \in \mathcal{D}\left(\mathcal{A}^{*}\right)
\end{gathered}
$$


Since $\varepsilon \in\left(\frac{1}{2}, 1\right]$, we have $\frac{1-\varepsilon}{2} \in\left[0, \frac{1}{4}\right)$. Thus, with [21, Theorem 3A.1], for the fractional powers of $\left(\lambda_{0} I-\mathcal{A}^{*}\right)$, it holds that

$$
\mathcal{D}\left(\left(\lambda_{0} I-\mathcal{A}^{*}\right)^{\frac{1-\varepsilon}{2}}\right)=\left[\mathcal{D}\left(\lambda_{0} I-\mathcal{A}^{*}\right), L^{2}(\Omega) \times L^{2}(\Omega)\right]_{\frac{1+\varepsilon}{2}}=H^{1-\varepsilon}(\Omega) \times L^{2}(\Omega) .
$$

Note that $1-\varepsilon \in\left[0, \frac{1}{2}\right)$ and, therefore, with [22, Theorem 11.1], we conclude that $H^{1-\varepsilon}(\Omega) \times L^{2}(\Omega)=H_{0}^{1-\varepsilon}(\Omega) \times L^{2}(\Omega)$. In particular, we also get

$$
\left(\mathcal{D}\left(\left(\lambda_{0} I-\mathcal{A}^{*}\right)^{\frac{1-\varepsilon}{2}}\right)\right)^{*}=\left(H^{1-\varepsilon}(\Omega) \times L^{2}(\Omega)\right)^{*}=H^{\varepsilon-1}(\Omega) \times L^{2}(\Omega) .
$$

Next, we use that $\frac{1+\varepsilon}{2} \in\left(\frac{3}{4}, 1\right]$. Following once more [21, Theorem 3A.1], this implies that

$$
\begin{aligned}
\mathcal{D}\left(\left(\lambda_{0} I-\mathcal{A}\right)^{\frac{1+\varepsilon}{2}}\right) & =\left[\mathcal{D}\left(\lambda_{0} I-\mathcal{A}\right), L^{2}(\Omega) \times L^{2}(\Omega)\right]_{\frac{1-\varepsilon}{2}} \\
& =H_{\nu}^{1+\varepsilon}(\Omega) \times L^{2}(\Omega):=\left\{y \in H^{1+\varepsilon}(\Omega) \times L^{2}(\Omega) \mid \frac{\partial y_{v}}{\partial \nu}=0 \text { on } \Gamma\right\} .
\end{aligned}
$$

Finally, from e.g. [20, Appendix B], we deduce

$$
\left[\mathcal{D}\left(\lambda_{0} I-\mathcal{A}^{*}\right), L^{2}(\Omega) \times L^{2}(\Omega)\right]_{\frac{1}{2}}=H^{1}(\Omega) \times L^{2}(\Omega) .
$$

Step 2: The semigroup generated by $\mathcal{A}_{\Pi}$ is analytic and exponentially stable on $L^{2}(\Omega) \times L^{2}(\Omega)$. Moreover, we know that

$$
\begin{aligned}
\vec{y}_{0} & \in H^{\varepsilon}(\Omega) \times H^{1+\varepsilon}(\Omega) \subset L^{2}(\Omega) \times L^{2}(\Omega) \subset\left(H^{1}(\Omega)\right)^{*} \times L^{2}(\Omega), \\
\vec{f} & \in L^{2}\left(0, \infty ; H^{\varepsilon-1}(\Omega)\right) \times L^{2}\left(Q_{\infty}\right) \subset L^{2}\left(0, \infty ;\left(\mathcal{D}\left(\mathcal{A}_{\Pi}^{*}\right)\right)^{*}\right) .
\end{aligned}
$$

Hence, we can use [32, Lemma 4.2] and (4.6) to show that the mapping

$$
\begin{aligned}
\vec{y} & \mapsto\left(\vec{y}^{\prime}-\mathcal{A}_{\Pi} \vec{y}, \vec{y}(0)\right) \\
\left(L^{2}\left(Q_{\infty}\right) \times L^{2}\left(Q_{\infty}\right)\right) \cap H^{1}\left(0, \infty ;\left(\mathcal{D}\left(\mathcal{A}_{\Pi}^{*}\right)\right)^{*}\right) & \rightarrow L^{2}\left(0, \infty ;\left(\mathcal{D}\left(\mathcal{A}_{\Pi}^{*}\right)\right)^{*}\right) \times\left[\mathcal{D}\left(\mathcal{A}_{\Pi}^{*}\right), L^{2}(\Omega) \times L^{2}(\Omega)\right]_{\frac{1}{2}}^{*}
\end{aligned}
$$

is an isomorphism. In particular, there exists a solution $\vec{y} \in L^{2}\left(Q_{\infty}\right) \times L^{2}\left(Q_{\infty}\right)$ satisfying

$$
\|\vec{y}\|_{L^{2}\left(Q_{\infty}\right) \times L^{2}\left(Q_{\infty}\right)} \leq C\left(\left\|y_{0}\right\|_{H^{\varepsilon}(\Omega) \times H^{1+\varepsilon}(\Omega)}+\|\vec{f}\|_{L^{2}\left(0, \infty ; H^{\varepsilon-1}(\Omega)\right) \times L^{2}\left(Q_{\infty}\right)}\right) .
$$

Step 3: Consider now the splitting $\vec{y}=\vec{y}_{1}+\vec{y}_{2}$, where

$$
\begin{aligned}
& \vec{y}_{1}^{\prime}=\left(\mathcal{A}-\lambda_{0} I\right) \vec{y}_{1}+\vec{f}+\lambda_{0} \vec{y}, \quad \vec{y}_{1}(0)=\vec{y}_{0}, \\
& \vec{y}_{2}^{\prime}=\left(\mathcal{A}-\lambda_{0} I\right) \vec{y}_{2}-\left(\begin{array}{c}
B B^{*} \Pi \vec{y}_{v} \\
0
\end{array}\right), \quad \vec{y}_{2}(0)=0,
\end{aligned}
$$

with $\vec{f}$ and $\vec{y}_{0}$ as above. From (4.7), we know that $\vec{y} \in L^{2}\left(Q_{\infty}\right) \times L^{2}\left(Q_{\infty}\right)$. Using (4.3) and (4.4), we get

$$
\begin{aligned}
\lambda_{0} \vec{y} \in L^{2}\left(Q_{\infty}\right) \times L^{2}\left(Q_{\infty}\right) & \subset L^{2}\left(0, \infty ; H^{\varepsilon-1}(\Omega)\right) \times L^{2}\left(Q_{\infty}\right), \\
\vec{f} \in L^{2}\left(0, \infty ; H^{\varepsilon-1}(\Omega)\right) \times L^{2}\left(Q_{\infty}\right) & =\left[\mathcal{D}\left(\lambda_{0} I-\mathcal{A}^{*}\right), L^{2}(\Omega) \times L^{2}(\Omega)\right]_{\frac{1+\varepsilon}{2}}^{*} \times L^{2}\left(Q_{\infty}\right) .
\end{aligned}
$$


Following [13, p. 171], for $A_{0}=\lambda_{0} I-\mathcal{A} \in \mathcal{L}\left(\mathcal{D}\left(A_{0}\right), L^{2}(\Omega) \times L^{2}(\Omega)\right), A_{1}=\lambda_{0} I-\mathcal{A}^{*} \in$ $\mathcal{L}\left(L^{2}(\Omega) \times L^{2}(\Omega),\left(\mathcal{D}\left(A_{0}\right)\right)^{*}\right), E_{0}=L^{2}(\Omega) \times L^{2}(\Omega)$ and $E_{1}=\left(\mathcal{D}\left(\lambda_{0} I-A\right)\right)^{*}$, it holds that

$$
\left[\left[\mathcal{D}\left(A_{0}\right), E_{0}\right]_{\frac{1-\varepsilon}{2}},\left[\mathcal{D}\left(A_{1}\right), E_{1}\right]_{\frac{1-\varepsilon}{2}}\right]_{\frac{1}{2}}=\left[\left[\mathcal{D}\left(A_{0}\right), \mathcal{D}\left(A_{1}\right)\right]_{\frac{1}{2}},\left[E_{0}, E_{1}\right]_{\frac{1}{2}}\right]_{\frac{1-\varepsilon}{2}} .
$$

Hence, with [22, Theorem 12.4] this implies

$$
\begin{aligned}
& {\left[H_{\nu}^{1+\varepsilon}(\Omega) \times L^{2}(\Omega), H^{\varepsilon-1}(\Omega) \times L^{2}(\Omega)\right]_{\frac{1}{2}}} \\
& =\left[\left[\mathcal{D}\left(\lambda_{0} I-\mathcal{A}\right), L^{2}(\Omega) \times L^{2}(\Omega)\right]_{\frac{1-\varepsilon}{2}},\left[\mathcal{D}\left(\lambda_{0} I-\mathcal{A}^{*}\right), L^{2}(\Omega) \times L^{2}(\Omega)\right]_{\frac{1+\varepsilon}{2}}^{*}\right]_{\frac{1}{2}} \\
& =\left[\left[\mathcal{D}\left(\lambda_{0} I-\mathcal{A}\right), L^{2}(\Omega) \times L^{2}(\Omega)\right]_{\frac{1}{2}},\left[L^{2}(\Omega) \times L^{2}(\Omega),\left(\mathcal{D}\left(\lambda_{0} I-\mathcal{A}^{*}\right)\right)^{*}\right]_{\frac{1}{2}}\right]_{\frac{1-\varepsilon}{2}} \\
& =\left[H^{1}(\Omega),\left(H^{1}(\Omega)\right)^{*}\right]_{\frac{1-\varepsilon}{2}}=H^{\varepsilon}(\Omega) .
\end{aligned}
$$

Combining the previous statement with

$$
\vec{y}_{1}(0)=\vec{y}_{0} \in H^{\varepsilon}(\Omega) \times H^{1+\varepsilon}(\Omega) \subset H^{\varepsilon}(\Omega) \times L^{2}(\Omega),
$$

we can apply Theorem 4.6 with $\alpha=\frac{1-\varepsilon}{2}$ to (4.8) in order to show the existence of a unique solution

$$
\vec{y}_{1} \in L^{2}\left(0, \infty ; H_{\nu}^{1+\varepsilon}(\Omega) \times L^{2}(\Omega)\right) \cap H^{1}\left(0, \infty ; H^{\varepsilon-1}(\Omega) \times L^{2}(\Omega)\right)
$$

to (4.8) satisfying

$$
\begin{aligned}
& \left\|\vec{y}_{1}\right\|_{L^{2}\left(0, \infty ; H_{\nu}^{1+\varepsilon}(\Omega) \times L^{2}(\Omega)\right) \cap H^{1}\left(0, \infty ; H^{\varepsilon-1}(\Omega) \times L^{2}(\Omega)\right)} \\
& \quad \leq C\left(\left\|y_{0}\right\|_{H^{\varepsilon}(\Omega) \times L^{2}(\Omega)}+\|\vec{f}\|_{L^{2}\left(0, \infty ; H^{\varepsilon-1}(\Omega)\right) \times L^{2}\left(Q_{\infty}\right)}+\|\vec{y}\|_{L^{2}\left(Q_{\infty}\right) \times L^{2}\left(Q_{\infty}\right)}\right) .
\end{aligned}
$$

Let us next focus on $\vec{y}_{2}$. It clearly holds that $B B^{*} \Pi \vec{y}_{v} \in L^{2}\left(Q_{\infty}\right)$. Hence, by [5, Proposition 3.7] the second equation in (4.8) has a unique solution

$$
\vec{y}_{2} \in L^{2}\left(0, \infty ; \mathcal{D}\left(\lambda_{0} I-\mathcal{A}\right)\right) \cap H^{1}\left(0, \infty ; L^{2}(\Omega) \times L^{2}(\Omega)\right) .
$$

For $\varepsilon \in\left(\frac{1}{2}, 1\right]$ this in particular leads to

$$
\vec{y}_{2} \in L^{2}\left(0, \infty ; H_{\nu}^{1+\varepsilon}(\Omega) \times L^{2}(\Omega)\right) \cap H^{1}\left(0, \infty ; H^{\varepsilon-1}(\Omega) \times L^{2}(\Omega)\right) .
$$

As a consequence, we conclude for $\vec{y}=\vec{y}_{1}+\vec{y}_{2}$, that

$$
\vec{y} \in L^{2}\left(0, \infty ; H_{\nu}^{1+\varepsilon}(\Omega) \times L^{2}(\Omega)\right) \cap H^{1}\left(0, \infty ; H^{\varepsilon-1}(\Omega) \times L^{2}(\Omega)\right) .
$$

Step 4: Up to now, we only know that $y_{w} \in H^{1}\left(0, \infty ; L^{2}(\Omega)\right)$. We still have to show that moreover $y_{w} \in C\left([0, \infty] ; H^{1+\varepsilon}(\Omega)\right) \cap H^{1}\left(0, \infty ; H^{1+\varepsilon}(\Omega)\right)$ holds. From (3.8), we have

$$
y_{w}^{\prime}=\gamma y_{v}-\delta y_{w}, y_{w}(0) \in H^{1+\varepsilon}(\Omega) .
$$

Hence, we get the explicit representation

$$
y_{w}(t)=e^{-\delta t} y_{w}(0)+\gamma \int_{0}^{t} e^{-\delta(t-\tau)} y_{v}(\tau) \mathrm{d} \tau .
$$


In particular, $y_{w}(t)$ is continuous with values in $H^{1+\varepsilon}(\Omega)$. Moreover, we get $\left\|y_{w}\right\|_{L^{2}\left(0, \infty ; H^{1+\varepsilon}(\Omega)\right)} \leq\left\|y_{w}(0)\right\|_{H^{1+\varepsilon}(\Omega)}\left(\int_{0}^{\infty} e^{-2 \delta t} \mathrm{~d} t\right)^{\frac{1}{2}}+\gamma\left\|e^{-\delta} *\right\| y_{v}\left\|_{H^{1+\varepsilon}(\Omega)}\right\|_{L^{2}[0, \infty]}$.

The first term clearly exists. Since $e^{-t} \in L^{1}[0, \infty]$ and $y_{v} \in L^{2}\left(0, \infty ; H^{1+\varepsilon}(\Omega)\right)$, for the second term, Young's inequality for convolutions gives $e^{-\delta} *\left\|y_{v}\right\|_{H^{1+\varepsilon}(\Omega)} \in L^{2}[0, \infty]$. Since $y_{v} \in L^{2}\left(0, \infty ; H^{1+\varepsilon}(\Omega)\right)$ as well as $y_{w} \in L^{2}\left(0, \infty ; H^{1+\varepsilon}(\Omega)\right)$, this implies $y_{w} \in$ $H^{1}\left(0, \infty ; H^{1+\varepsilon}(\Omega)\right)$ by $(4.9)$.

Step 5: Finally, we show that $y_{v} \in H^{1+\varepsilon, \frac{1+\varepsilon}{2}}\left(Q_{\infty}\right)$. For $\varepsilon=1$, this is clear. Moreover, from [22, p. 47], for two Hilbert spaces $X$ and $Y$ and scalars $s_{1}, s_{2} \in \mathbb{R}, \theta \in(0,1)$, we have

$$
\left[H^{s_{1}}(0, \infty ; X), H^{s_{2}}(0, \infty ; Y)\right]_{\theta}=H^{(1-\theta) s_{1}+\theta s_{2}}\left(0, \infty ;[X, Y]_{\theta}\right) .
$$

Since $y_{v} \in L^{2}\left(0, \infty ; H_{\nu}^{1+\varepsilon}(\Omega)\right) \cap H^{1}\left(0, \infty ; H^{\varepsilon-1}(\Omega)\right)$, we can choose $\theta=\frac{1+\varepsilon}{2}, s_{1}=$ $0, s_{2}=1, X=H^{1+\varepsilon}(\Omega)$ and $Y=H^{\varepsilon-1}(\Omega)$, and conclude that $y_{v} \in H^{\frac{1+\varepsilon}{2}}\left(0, \infty ; L^{2}(\Omega)\right)$. (

The second main result is the local stabilization of the nonlinear closed-loop system.

THEOREM 4.8. Let $\Omega \subset \mathbb{R}^{2}$ and $\varepsilon \in\left(\frac{1}{2}, 1\right]$. Then there exist $\mu_{0}>0$ and a nondecreasing function $\eta$ from $\mathbb{R}^{+}$into itself, such that if $\mu \in\left(0, \mu_{0}\right)$ and $\left\|\vec{y}_{0}\right\|_{H^{\varepsilon}(\Omega) \times H^{1+\varepsilon}(\Omega)} \leq$ $\eta(\mu)$, then

$$
\vec{y}^{\prime}=\mathcal{A} \vec{y}+\mathcal{F}(\vec{y}), \quad \vec{y}(0)=\vec{y}_{0},
$$

admits a unique solution in the set

$$
\begin{array}{r}
D_{\mu}=\left\{\vec{y} \in H^{1+\varepsilon, \frac{1+\varepsilon}{2}}\left(Q_{\infty}\right) \times\left(C\left([0, \infty] ; H^{1+\varepsilon}(\Omega)\right) \cap H^{1}\left(0, \infty ; H^{1+\varepsilon}(\Omega)\right)\right),\right. \\
\left.\|\vec{y}\|_{H^{1+\varepsilon, \frac{1+\varepsilon}{2}}\left(Q_{\infty}\right) \times H^{1}\left(0, \infty ; H^{1+\varepsilon}(\Omega)\right)} \leq \mu\right\} .
\end{array}
$$

Proof. With the results established so far, we can now utilize a fixed point argument as in [31, Theorem 5.1]. We show that the mapping $\mathcal{M}: \vec{z} \mapsto \vec{y}_{z}$ defined by

$$
\vec{y}^{\prime}=\mathcal{A}_{\Pi} \vec{y}+\mathcal{F}(z), \vec{y}(0)=\vec{y}_{0},
$$

is a contraction in $D_{\mu}$. For this, we set

$$
\mu_{0}=\frac{1}{2}\left(\sqrt{1+\frac{1}{C_{1} C_{2}}}-1\right) \text { and } \eta(\mu)=\frac{3}{4 C_{1}} \mu,
$$

with $C_{1}$ and $C_{2}$ as in, respectively, Lemma 4.4 and Theorem 4.7.

Step 1: For $\vec{z} \in D_{\mu}$, from Lemma 4.4 we get

$$
\|\mathcal{F}(\vec{z})\|_{L^{2}\left(0, \infty ; H^{\varepsilon-1}(\Omega)\right) \times L^{2}\left(Q_{\infty}\right)} \leq C_{1}\left(\mu+\mu^{2}\right) \mu .
$$

Moreover, with Theorem 4.7 it follows that

$$
\begin{aligned}
& \left\|\vec{y}_{z}\right\|_{H^{1+\varepsilon}, \frac{1+\varepsilon}{2}}(\Omega) \times H^{1}\left(0, \infty ; H^{1+\varepsilon}(\Omega)\right) \\
& \quad \leq C_{2}\left(\left\|y_{0}\right\|_{H^{\varepsilon}(\Omega) \times H^{1+\varepsilon}(\Omega)}+\|\mathcal{F}(\vec{z})\|_{L^{2}\left(0, \infty ; H^{\varepsilon-1}(\Omega)\right) \times L^{2}\left(Q_{\infty}\right)}\right) \\
& \quad \leq \frac{3}{4} \mu+C_{1} C_{2}\left(\mu+\mu^{2}\right) \mu .
\end{aligned}
$$


Now since $\mu \leq \mu_{0}=\frac{1}{2}\left(\sqrt{1+\frac{1}{C_{1} C_{2}}}-1\right)$, it also holds $\mu+\mu^{2} \leq \frac{1}{4 C_{1} C_{2}}$ and thus

$$
\left\|\vec{y}_{z}\right\|_{H^{1+\varepsilon, \frac{1+\varepsilon}{2}}(\Omega) \times H^{1}\left(0, \infty ; H^{1+\varepsilon}(\Omega)\right)} \leq \mu,
$$

particularly showing that $\mathcal{M}$ is mapping $D_{\mu}$ to itself.

Step 2: For $\vec{z}_{1}, \vec{z}_{2} \in D_{\mu}$ we further have

$$
\vec{y}_{1}^{\prime}-\vec{y}_{2}^{\prime}=\mathcal{A}_{\Pi}\left(\vec{y}_{1}-\vec{y}_{2}\right)+\mathcal{F}\left(\vec{z}_{1}\right)-\mathcal{F}\left(\vec{z}_{2}\right), \quad \vec{y}_{1}(0)-\vec{y}_{2}(0)=0
$$

Hence, Theorem 4.7 implies that

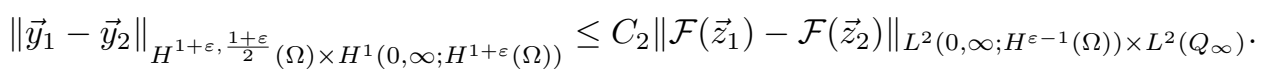

Using Lemma 4.4, this yields

$$
\begin{aligned}
\left\|\vec{y}_{1}-\vec{y}_{2}\right\|_{H^{1+\varepsilon, \frac{1+\varepsilon}{2}}(\Omega) \times H^{1}\left(0, \infty ; H^{1+\varepsilon}(\Omega)\right)} & \leq C_{1} 2\left(\mu+\mu^{2}\right)\left\|z_{1, v}-z_{2, v}\right\|_{1+\varepsilon, \frac{1+\varepsilon}{2}} \\
& \leq \frac{1}{2}\left\|z_{1, v}-z_{2, v}\right\|_{1+\varepsilon, \frac{1+\varepsilon}{2}}
\end{aligned}
$$

In other words, the mapping $\mathcal{M}$ is a contraction in $D_{\mu}$ and the proof is complete.

If $\varepsilon=1$, we have an analogous result for the three-dimensional case. Since the proof uses exactly the same arguments as above, we only state the final result.

THEOREM 4.9. Let $\Omega \subset \mathbb{R}^{3}$. Then there exist $\mu_{0}>0$ and a nondecreasing function $\eta$ from $\mathbb{R}^{+}$into itself, such that if $\mu \in\left(0, \mu_{0}\right)$ and $\left\|\vec{y}_{0}\right\|_{H^{1}(\Omega) \times H^{2}(\Omega)} \leq \eta(\mu)$, then

$$
\vec{y}^{\prime}=\mathcal{A} \vec{y}+\mathcal{F}(\vec{y}), \quad \vec{y}(0)=\vec{y}_{0}
$$

admits a unique solution in the set

$$
\begin{gathered}
D_{\mu}=\left\{\vec{y} \in H^{2,1}\left(Q_{\infty}\right) \times\left[C\left([0, \infty] ; H^{2}(\Omega)\right) \cap H^{1}\left(0, \infty ; H^{2}(\Omega)\right)\right]\right. \\
\left.\|\vec{y}\|_{H^{2,1}\left(Q_{\infty}\right) \times H^{1}\left(0, \infty ; H^{2}(\Omega)\right)} \leq \mu\right\}
\end{gathered}
$$

4.3. Exponential stabilization. Following [30, 32] we can also achieve stabilization with a prescribed exponential decay rate $\sigma<\delta$. For this, we set $\hat{\vec{y}}=e^{\sigma t} \vec{y}$ and $\hat{u}=e^{\sigma t} u$. Now, if $\vec{y}=\left(y_{v}, y_{w}\right)$ solves

$$
\begin{aligned}
& \frac{\partial y_{v}}{\partial t}=\Delta y_{v}-\left(3 a \bar{v}^{2}+2 b \bar{v}+c\right) y_{v}-d y_{w}-a y_{v}^{3}-(b+3 a \bar{v}) y_{v}^{2}+B u \\
& \frac{\partial y_{w}}{\partial t}=\gamma y_{v}-\delta y_{w}, \quad \vec{y}(0)=\vec{y}_{0}
\end{aligned}
$$

then $\hat{\vec{y}}=\left(\hat{y}_{v}, \hat{y}_{w}\right)$ solves

$$
\begin{aligned}
& \frac{\partial \hat{y}_{v}}{\partial t}=\Delta \hat{y}_{v}+\sigma \hat{y}_{v}-\left(3 a \bar{v}^{2}+2 b \bar{v}+c\right) \hat{y}_{v}-d \hat{y}_{w}-a e^{-2 \sigma t} \hat{y}_{v}^{3}-(b+3 a \bar{v}) e^{-\sigma t} \hat{y}_{v}^{2}+B \hat{u}, \\
& \frac{\partial \hat{y}_{w}}{\partial t}=\gamma \hat{y}_{v}-\delta \hat{y}_{w}+\sigma \hat{y}_{w}, \quad \hat{\vec{y}}(0)=\vec{y}_{0} .
\end{aligned}
$$


In order to stabilize the linearized system, we then have to solve the shifted Riccati equation

$$
(\mathcal{A}+\sigma \mathcal{I})^{*} \Pi_{\sigma}+\Pi_{\sigma}(\mathcal{A}+\sigma \mathcal{I})-\Pi_{\sigma} \mathcal{B} \mathcal{B}^{*} \Pi_{\sigma}+\mathcal{I}=0 .
$$

Alternatively to (4.15) we can again utilize the results from Lemma 3.2 for $\sigma<\delta$ and solve the Riccati equation for the decoupled system with $\alpha=\sigma+\gamma \frac{|d|}{\delta-\sigma}$. Since the Lipschitz properties shown in Lemma 4.4 and 4.5 similarly hold true for

$$
\hat{\mathcal{F}}(\hat{\vec{y}})=\left(\begin{array}{c}
-e^{-2 \sigma t} a \hat{y}_{v}^{3}-e^{-\sigma t}(b+3 a \bar{v}) \hat{y}_{v}^{2} \\
0
\end{array}\right)
$$

with the same arguments as before, we can show the stabilization of (4.14) and, hence, the exponential stabilization of (4.13). At this point, we refrain from a more detailed discussion and instead refer to the original idea proposed in [30].

5. Numerical examples. For the numerical simulations, we consider a finite difference discretization of the monodomain equations with the FitzHugh-Nagumo model for the ionic current. The precise setup is the following

$$
\begin{aligned}
\frac{\partial v}{\partial t} & =\sigma \Delta v-\eta_{0} v-\eta_{1} w+f(v)+g_{v}(x, y)+B u & & \text { in } \Omega \times(0, T), \\
\frac{\partial w}{\partial t} & =\frac{\eta_{2}}{v_{p k}} v-\eta_{2} \eta_{3} w, & & \text { in } \Omega \times(0, T), \\
\frac{\partial v}{\partial \nu} & =0, & & \text { on } \Gamma \times(0, T), \\
v(x, 0) & =v_{0}(x) \text { and } w(x, 0)=w_{0}(x), & & \text { in } \Omega,
\end{aligned}
$$

where $\Omega=(0,1) \times(0,1)$ and $f(v)=\eta_{0}\left(\frac{1}{v_{t h}}+\frac{1}{v_{p k}}\right) v^{2}-\frac{\eta_{0}}{v_{t h} v_{p k}} v^{3}$. The parameters are $\sigma=0.0015, \eta_{0}=1.5, \eta_{1}=215.6, \eta_{2}=0.012, \eta_{3}=1$. The threshold and peak potentials, respectively, are given as $v_{t h}=13$ and $v_{p k}=100$. All values are inspired by [17] where numerical experiments have been carried out for the Rogers-McCulloch model. For all examples, we use second order central differences for the Laplacian as well as for the homogeneous Neumann boundary conditions. The simulation domain is approximated by a uniform grid of size $h_{x}=h_{y}=\frac{1}{n_{x}-1}$ with $n_{x}=n_{y}=64$ grid points. The resulting semi-discrete finite dimensional ODE system is denoted by

$$
\begin{aligned}
\dot{v}_{n}(t) & =A_{11} v_{n}(t)+A_{12} w_{n}(t)+f\left(v_{n}(t)\right)+g_{v_{n}}+B_{n} u(t), \\
\dot{w}_{n}(t) & =A_{12} v_{n}(t)+A_{22} w_{n}(t),
\end{aligned}
$$

where $A_{11}, A_{12}, A_{21}, A_{22} \in \mathbb{R}^{n \times n}, B_{n} \in \mathbb{R}^{n \times m}, g_{v_{n}} \in \mathbb{R}^{n}$ and $n=n_{x} \cdot n_{y}=4096$.

All simulations are generated on an Intel ${ }^{\circledR} \mathrm{Core}^{\mathrm{TM}} \mathrm{i} 5-2500$ CPU, 8 GB RAM,

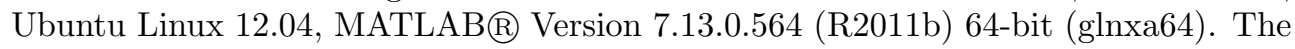
solutions of the ODE systems are always obtained by the MATLAB routine ode 45 .

5.1. A nonzero steady state. The first example we study is the stabilization of a constant stationary solution to (5.1) without external force $g_{v}(x, y)$. For this, we consider the underlying ODE system

$$
\begin{aligned}
& 0=\eta_{0} v+\eta_{1} w-\eta_{0}\left(\frac{1}{v_{t h}}+\frac{1}{v_{p k}}\right) v^{2}+\frac{\eta_{0}}{v_{t h} v_{p k}} v^{3}, \\
& 0=\frac{\eta_{2}}{v_{p k}} v-\eta_{2} \eta_{3} w .
\end{aligned}
$$


and can compute three equilibria

$$
\begin{aligned}
& \bar{v}_{1}=0, \quad \bar{v}_{2,3}=\frac{v_{p k}+v_{t h}}{2} \pm \frac{1}{2} \sqrt{\left(v_{p k}-v_{t h}\right)^{2}-4 \frac{\eta_{1} v_{t h}}{\eta_{0} \eta_{3}}}, \\
& \bar{w}_{1}=0, \quad \bar{w}_{2,3}=\frac{1}{v_{p k} \eta_{3}} \bar{v}_{2,3} .
\end{aligned}
$$

For the above chosen parameter values, one further obtains that the only unstable equilibrium is given as

$$
\bar{v}=\frac{v_{p k}+v_{t h}}{2}-\frac{1}{2} \sqrt{\left(v_{p k}-v_{t h}\right)^{2}-4 \frac{\eta_{1} v_{t h}}{\eta_{0} \eta_{3}}} \approx 51.63, \quad \bar{w} \approx 0.5163 .
$$

We now slightly perturb the constant state as follows

$$
\bar{v}_{0}(x, y)=\bar{v}+3 \cdot \sin (2 \pi x) \cdot \sin (4 \pi y), \quad \bar{w}_{0}(x, y)=\bar{w}+\frac{3}{100} \cdot \sin (2 \pi x) \cdot \sin (4 \pi y) .
$$

The resulting initial state for our numerical tests is shown in Figure 5.1a. For the control domain we choose (see also Figure 5.1b)

$$
\begin{aligned}
\Omega_{c o n} & =\Omega_{c o n, 1} \cup \Omega_{c o n, 2} \cup \Omega_{c o n, 3} \cup \Omega_{c o n, 4}, \\
\Omega_{c o n, 1} & =\left\{x, y \in(0,1):(x-0.25)^{2}+(y-0.25)^{2} \leq 0.04\right\}, \\
\Omega_{c o n, 2} & =\left\{x, y \in(0,1):(x-0.25)^{2}+(y-0.75)^{2} \leq 0.04\right\}, \\
\Omega_{c o n, 3} & =\left\{x, y \in(0,1):(x-0.75)^{2}+(y-0.25)^{2} \leq 0.04\right\}, \\
\Omega_{c o n, 4} & =\left\{x, y \in(0,1):(x-0.75)^{2}+(y-0.75)^{2} \leq 0.04\right\} .
\end{aligned}
$$

The approximation of the control operator $B$ thus is a restriction of the identity matrix to the control domain. In particular, we obtain $B_{n} \in \mathbb{R}^{4096 \times 1992}$. In order to validate the results from Theorem 4.8, we solve the nonlinear closed-loop system

$$
\begin{aligned}
\dot{v}_{n}(t) & =A_{11} v_{n}(t)+A_{12} w_{n}(t)+f\left(v_{n}(t)\right)-B_{n} B_{n}^{T} P_{n}\left(v_{n}(t)-\bar{v}\right), & & v_{n}(0)=\bar{v}_{0}, \\
\dot{w}_{n}(t) & =A_{12} v_{n}(t)+A_{22} w_{n}(t), & & w_{n}(0)=\bar{w}_{0},
\end{aligned}
$$

where $P_{n}$ is the solution to the algebraic matrix Riccati equation

$$
\left(A_{11}+\frac{\partial f}{\partial v_{n}}(\bar{v})+10 \cdot I_{n}\right)^{T} P_{n}+P_{n}\left(A_{11}+\frac{\partial f}{\partial v_{n}}(\bar{v})+10 \cdot I_{n}\right)-P_{n} B_{n} B_{n}^{T} P_{n}+I_{n}=0 .
$$

Some remarks are in order. We choose a shift of $10 \cdot I_{n}$ in order to guarantee a sufficient decay rate for the decoupled closed-loop system. We carried out analogous computations for smaller shifts but in these cases, we had to decrease the norm of the initial perturbation. From a numerical point of view, the massive number (1992) of inputs of course might prevent from using approximate low rank techniques for solving the Riccati equation. However, here we are rather interested in the general concept than in the efficient numerical solution of the Riccati equation. Moreover, already the matrix $A_{11}+\frac{\partial f}{\partial v_{n}}(\bar{v})$, representing the linearization of the constant steady state has 41 eigenvalues in the right complex half-plane. The shifted matrix for which we solve the Riccati equation has 339 unstable eigenvalues.

We compare the evolution of the uncontrolled with the evolution of the closedloop nonlinear system. As can be seen from Figure 5.1c, the feedback stabilizes the perturbed initial condition around the constant steady state $(\bar{v}, \bar{w})$. On the other hand, without control, the system quickly converges to the (stable) zero steady state explaining the almost constant $L^{2}$-error. 


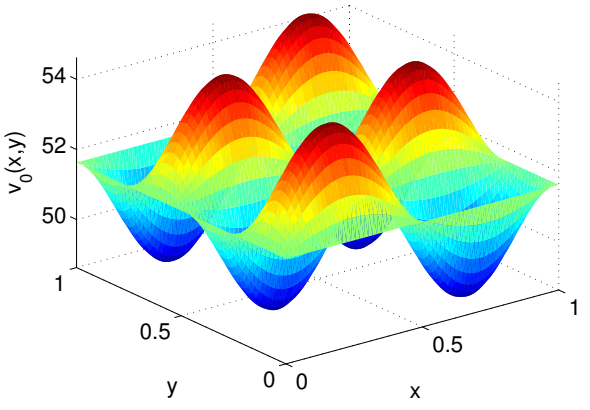

(a) Initial condition.

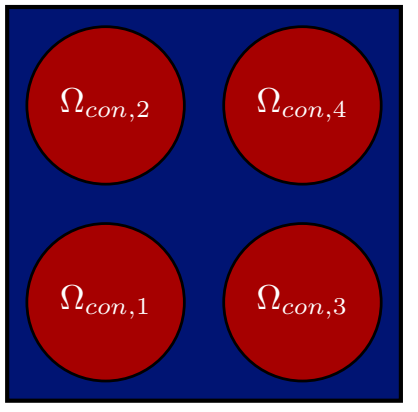

(b) Control domain.

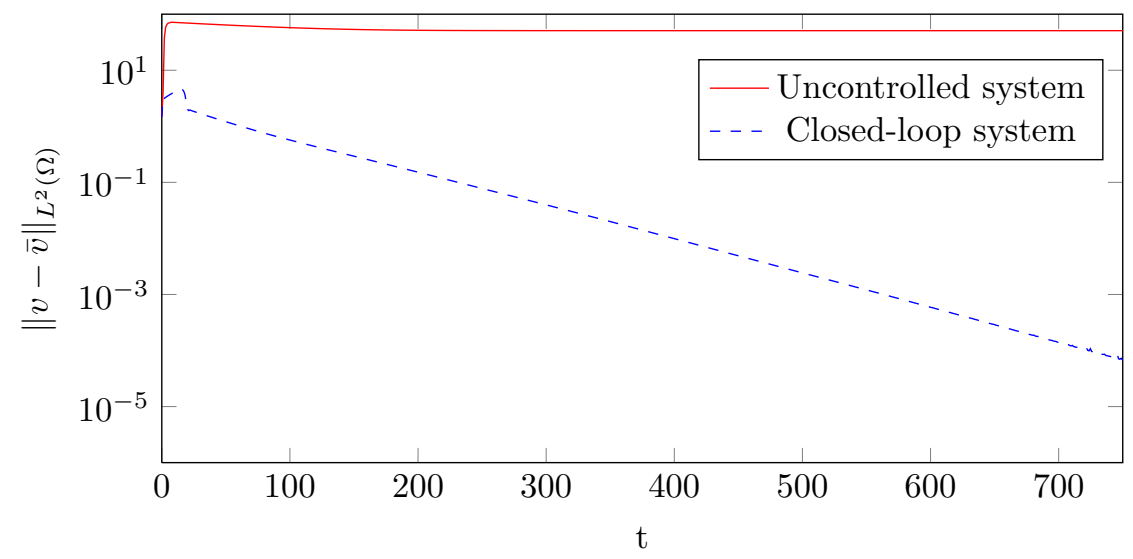

(c) $L^{2}$-error.

Fig. 5.1: A nonzero steady state.

5.2. Steady state with external force. Our second test case includes an additional external force $g_{v}(x, y)$. The term $g_{v}$ is chosen such that

$$
\begin{aligned}
& \bar{v}(x, y)=100\left(\frac{1}{2} x^{2}-\frac{1}{3} x^{3}\right)-50\left(\frac{1}{2} y^{2}-\frac{1}{3} y^{3}\right), \\
& \bar{w}(x, y)=\frac{1}{\eta_{3} v_{p k}} \bar{v}(x, y),
\end{aligned}
$$

is a steady state solution to (5.1). Of course, we can get an analytic expression for $g_{v}$ by evaluating

$$
g_{v}=-\sigma \Delta \bar{v}+\eta_{0} \bar{v}+\frac{\eta_{1}}{v_{p k} \eta_{3}} \bar{v}-f(\bar{v}) .
$$

The steady state again is chosen such that the system is unstable. We have three unstable eigenvalues for the linearized system matrix $A_{11}+\frac{\partial f}{\partial v_{n}}(\bar{v})$ and 296 unstable eigenvalues for $A_{11}+\frac{\partial f}{\partial v_{n}}(\bar{v})+12 \cdot I_{n}$. The latter matrix is used for computing the feedback gain via solving the associated matrix Riccati equation. 


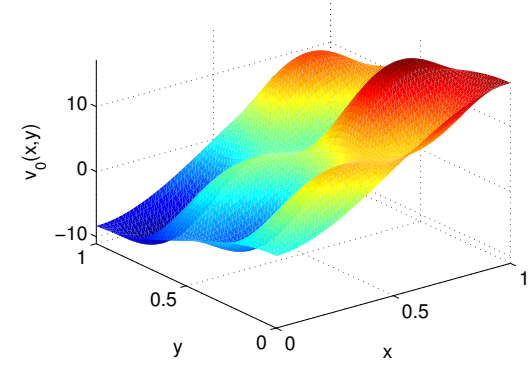

(a) Initial condition.

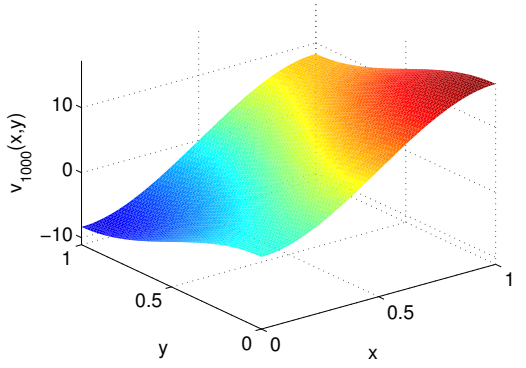

(b) Final state.

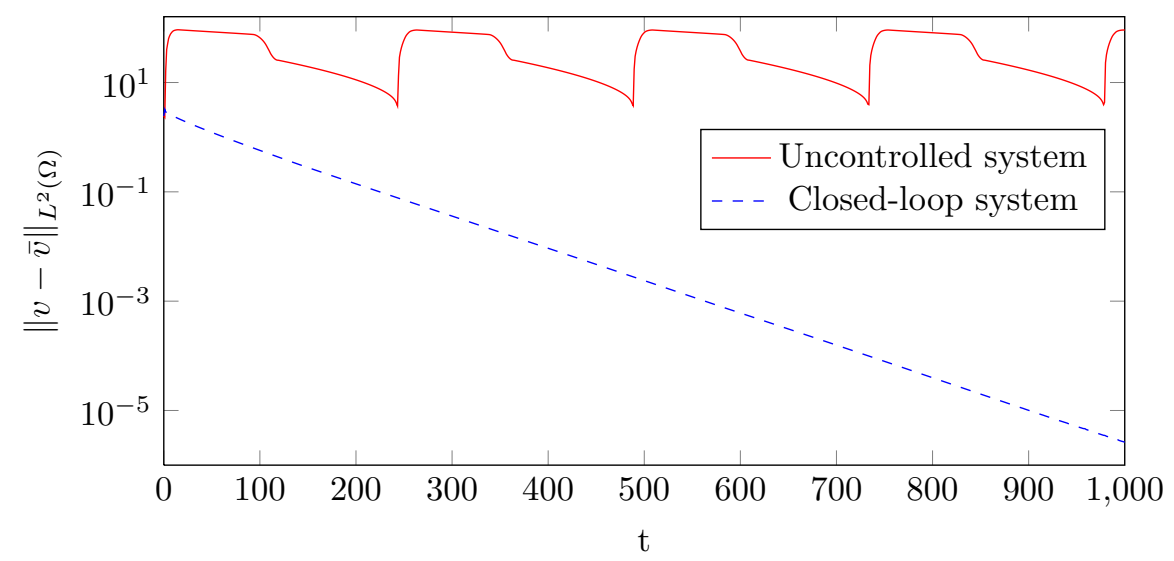

(c) $L^{2}$-error.

Fig. 5.2: Steady state with external force.

It is worthwhile to mention that even when initialized with the exact initial condition, the uncontrolled system will deviate from $(\bar{v}, \bar{w})$. This is simply due to the fact that the discretization of the analytically computed external force does not match with the external force for the discretized system, i.e.,

$$
g_{v, n} \neq-A_{11} \bar{v}-A_{12} \bar{w}-f(\bar{v}) .
$$

For this reason, instead of $g_{v, n}$ we use the latter expression in order to guarantee steady state behavior for the discretization of (5.4). The control domain now is chosen as in the first example. Similarly, we perturb the initial state by a trigonometric term as follows

$$
\bar{v}_{0}(x, y)=\bar{v}+5 \cdot \sin (2 \pi x) \cdot \sin (4 \pi y), \quad \bar{w}_{0}(x, y)=\bar{w}+\frac{5}{100} \cdot \sin (2 \pi x) \cdot \sin (4 \pi y) .
$$

Figure 5.2 and Figure 5.2b now show the initial as well as the final state $(t=1000)$ of the nonlinear closed-loop system. Again, we find that the linear feedback law stabilizes the nonlinear system, see also Figure 5.2c. In contrast to the first example, the uncontrolled system does not approach a constant (in time) $L^{2}$-error but exhibits 


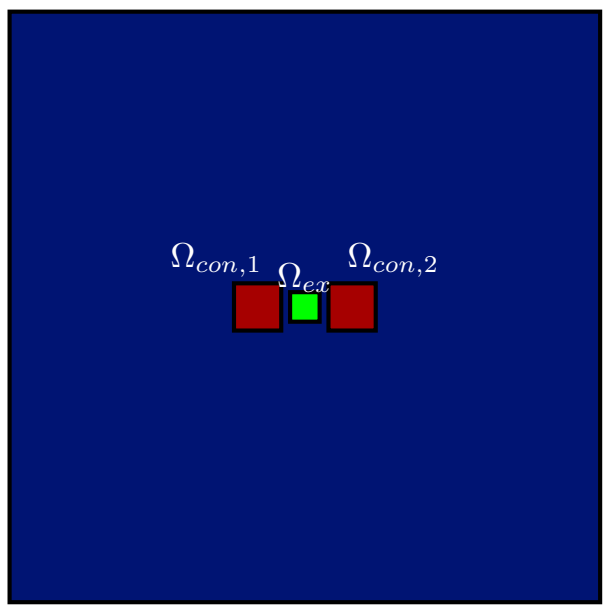

Fig. 5.3: Control domain and external stimulus.

a periodic behavior. Note that due to the external forcing term, the trivial solution no longer is a steady state of the equation.

5.3. Termination of an excitation wave. The final two examples should be understood as a phenomenological proof of concept for a possible increase of the stability radius by solving the Riccati equation. With regard to this situation, our goal is to control a given initial state to zero. From a practical point of view this case is relevant since the monodomain equations model the electric potential of the heart tissue. In this medical context it is usually desirable to reach the zero state. We begin with the prototypical test case of an excitation wave, see, e.g., [17]. Here, the initial condition describes a small part of the domain that gets excited by an external stimulus. We model this by setting

$$
\bar{v}_{0}(x, y)=101, \text { for }(x, y) \in[0.475,0.525] \times[0.475,0.525] .
$$

All other parts of the domain are at rest. For the external force it holds that $g_{v}=0$. As a result, for the uncontrolled system an excitation wave appears and the entire domain gets excited. After a certain period of time, the wave collapses and the system approaches the zero state. In a real-life context, one clearly wants to avoid this behavior and is interested in terminating the excitation wave as fast as possible.

In our example, the control domain is smaller than in the first two cases. However, in order to terminate the wave sufficiently fast, the actuators have to be close to the external stimulus. More precisely, we use (see Figure 5.3),

$$
\begin{aligned}
\Omega_{c o n} & =\Omega_{c o n, 1} \cup \Omega_{c o n, 2}, \\
\Omega_{c o n, 1} & =\{x, y \in[0.38,0.46] \times[0.46,0.54]\}, \\
\Omega_{c o n, 2} & =\{x, y \in[0.54,0.62] \times[0.46,0.54]\} .
\end{aligned}
$$

We obtain a discrete approximation of the control operator $B_{n} \in \mathbb{R}^{4096 \times 72}$. The gain matrix $R^{-1} B_{n}^{T} P_{n}$ is obtained by the solution of the matrix Riccati equation

$$
A_{11}^{T} P_{n}+P_{n} A_{11}-P_{n} B_{n} R^{-1} B_{n}^{T} P_{n}+I_{n}=0,
$$




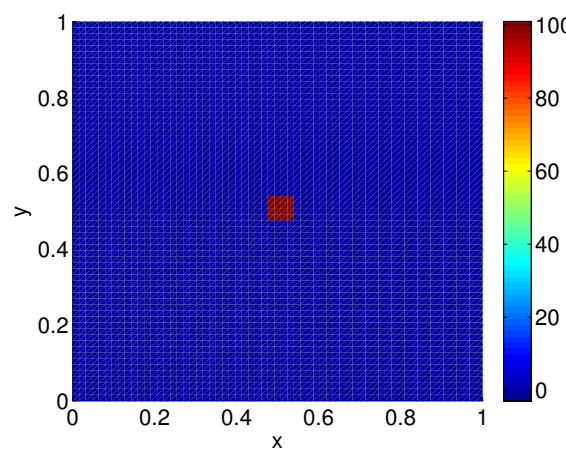

(a) $v(x, y)$ at $t=0$.

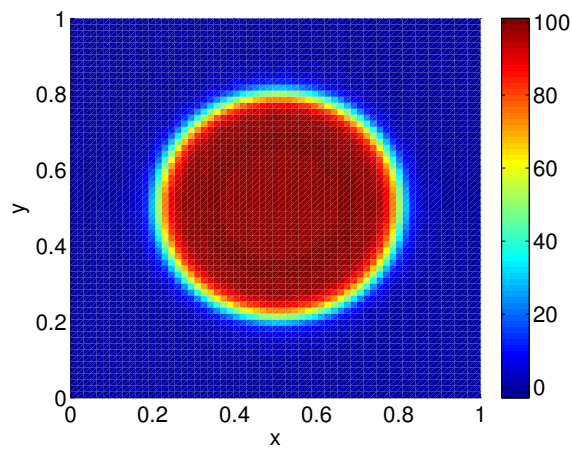

(c) $v(x, y)$ at $t=5$.

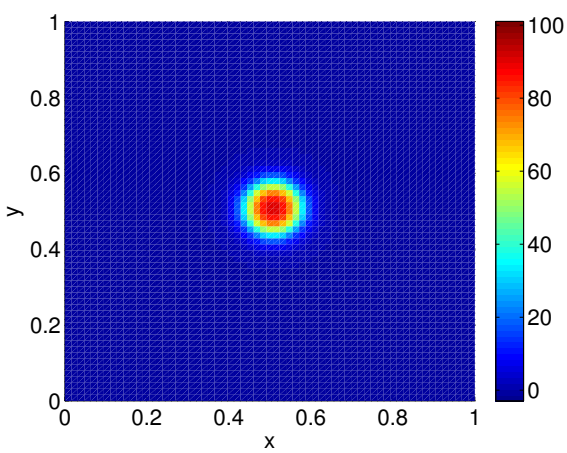

(b) $v(x, y)$ at $t=1$.

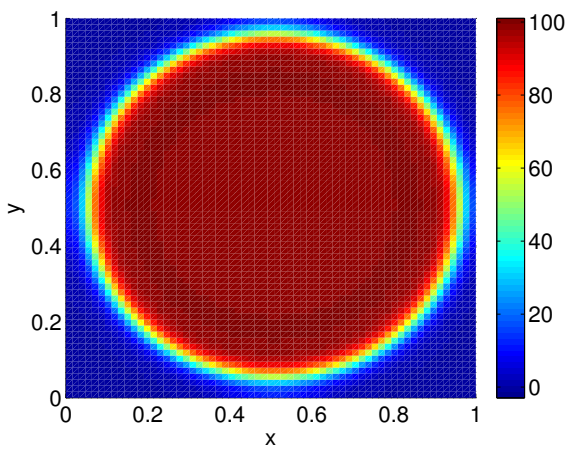

(d) $v(x, y)$ at $t=7.5$.

Fig. 5.4: Uncontrolled excitation wave.

where we choose the weight matrix $R=10^{-2} \cdot I_{n}$. In Figure 5.4 we show the evolution of the uncontrolled system for different time steps. As we already mentioned, one can see a quick excitation of the entire domain. On the other hand, the feedback controller counteracts with the external stimulus and, as a consequence, terminates the excitation wave after a very small period of time. We recall that this phenomenon is not covered by our stabilization result from Theorem 4.8. Theoretically we could have decreased the stability radius by solving the Riccati equation.

5.4. Termination of a reentry wave. Another important example is the termination of a so-called reentry wave that can be used as a model for fibrillation processes of the human heart. In order to generate an initial state developing into such a wave, we excite the transmembrane potential at the lower boundary of the domain with an external stimulus $S_{1, e x t}=101$. As a result, a traveling wave appears and moves through the domain. After a certain period of time (when the wave has disappeared; in our case $t=189.5$ ), we use a second stimulus of the form

$$
S_{2, e x t}=200 \text { in } \Omega_{e x t}=\left\{x, y \in(0,1): 4(x-0.5)^{2}+(y-0.5)^{2} \leq 0.38^{2}\right\},
$$

to excite the tissue for a small time interval $(t=2)$. Since reentry waves do not occur for all parameter settings, the previous setup has been determined experimentally. 


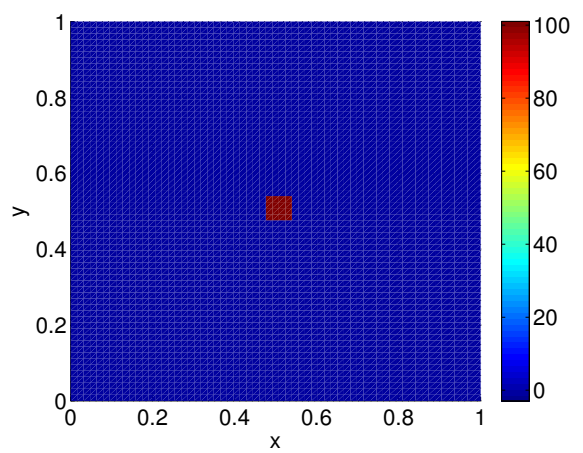

(a) $v(x, y)$ at $t=0$.

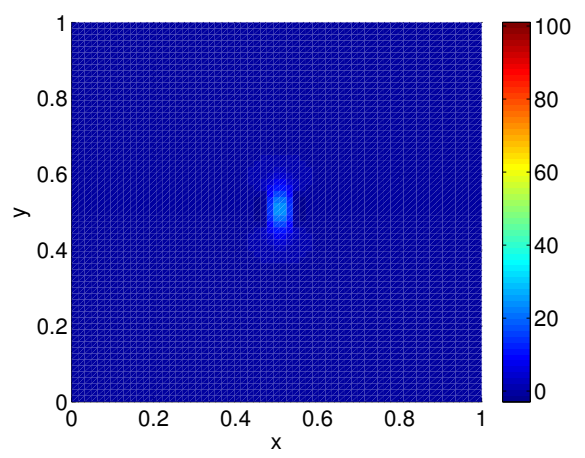

(c) $v(x, y)$ at $t=1$.

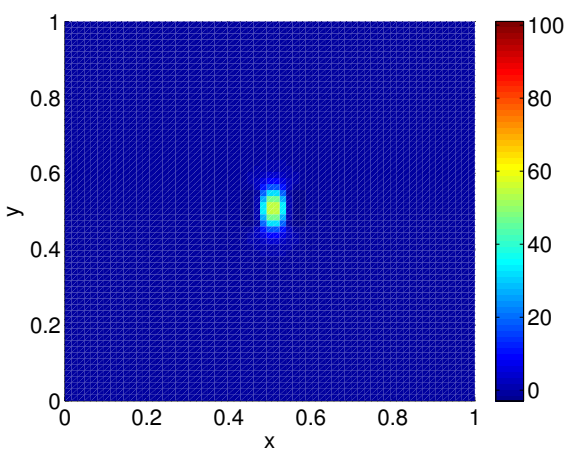

(b) $v(x, y)$ at $t=0.5$.

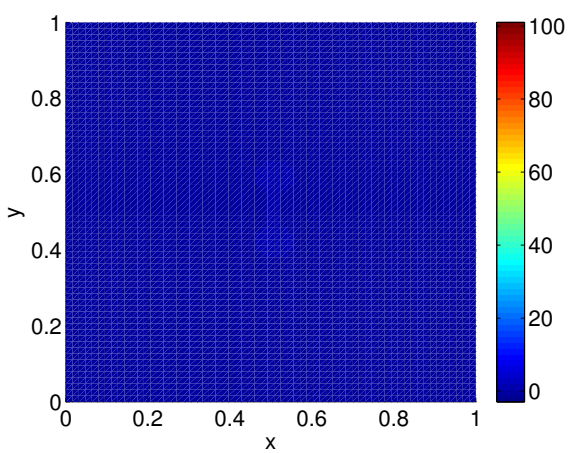

(d) $v(x, y)$ at $t=2$.

Fig. 5.5: Termination of excitation wave.

In Figure 5.7a we show the initial condition for the uncontrolled as well as for the closed-loop system. In this example, we stay close to a realistic setup where the control usually is located at (or near) the boundary of the tissue. The control domain is shown in Figure 5.6 and is given as

$$
\begin{aligned}
\Omega_{c o n} & =\Omega_{c o n, 1} \cup \Omega_{c o n, 2} \cup \Omega_{c o n, 3} \cup \Omega_{c o n, 4}, \\
\Omega_{c o n, 1} & =[0.015,0.125] \times[0.015,0.495], \\
\Omega_{c o n, 2} & =[0.015,0.125] \times[0.505,0.985], \\
\Omega_{c o n, 3} & =[0.875,0.985] \times[0.015,0.495], \\
\Omega_{c o n, 4} & =[0.875,0.985] \times[0.505,0.985] .
\end{aligned}
$$

In contrast to the other examples, we localize the control on each of the subdomains such that for the approximate control operator we have $B_{n} \in \mathbb{R}^{4096 \times 4}$. Again, the gain matrix $R^{-1} B_{n}^{T} P_{n}$ is obtained by solving

$$
A_{11}^{T} P_{n}+P_{n} A_{11}-P_{n} B_{n} R^{-1} B_{n}^{T} P_{n}+I_{n}=0,
$$

with weight matrix $R=10^{-2} \cdot I_{n}$. 


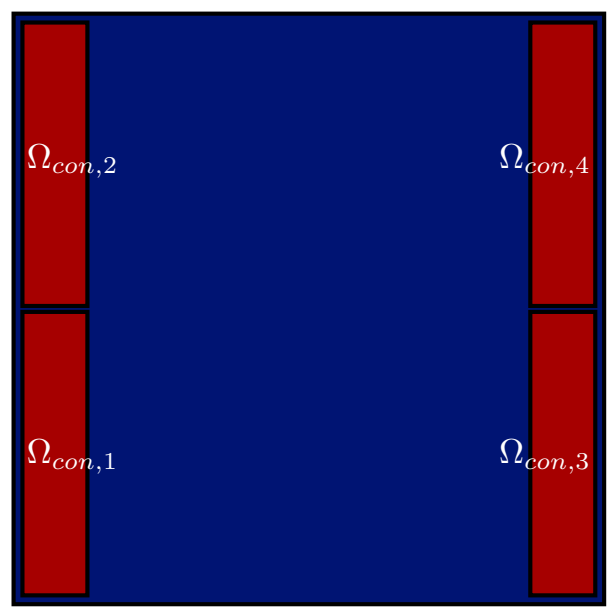

Fig. 5.6: Control domain for reentry wave.

As can be seen in Figure 5.7, the uncontrolled system describes the explained reentry behavior and does never come to rest. On the other hand, the results in Figure 5.8 indicate the positive effect of the feedback law, resulting in a termination of the reentry wave.

Acknowledgement. The authors gratefully acknowledge the Austrian Science Fund (FWF) for financial support under SFB F32, "Mathematical Optimization and Applications in Biomedical Sciences."

\section{REFERENCES}

[1] M. BADRA, Stabilisation par feedback et approximation des équations de Navier-Stokes, $\mathrm{PhD}$ thesis, Université Paul Sabatier, Toulouse, France, 2006.

[2] V. BARBU, Feedback stabilization of Navier-Stokes equations, ESAIM: Control, Optimisation and Calculus of Variations, 9 (2003), pp. 197-205.

[3] V. BARBu AND G. WANG, Feedback stabilization of semilinear heat equations, in Abstract and Applied Analysis, no. 12, Hindawi Publishing Corporation, 2003, pp. 697-714.

[4] - Internal stabilization of semilinear parabolic systems, Journal of mathematical analysis and applications, 285 (2003), pp. 387-407.

[5] A. Bensoussan, G. Da Prato, M.C. Delfour, and S.K. Mitter, Representation and control of infinite dimensional systems, vol. 2, Birkhäuser Boston, 1993.

[6] A.J.B. Brandão, E. Fernández-Cara, P.M.D. Magalhães, and M.A. Rojas-Medar, Theoretical analysis and control results for the FitzHugh-Nagumo equation, Electronic Journal of Differential Equations, 2008 (2008), pp. 1-20.

[7] R. Buchholz, H. Engel, E. Kammann, and F. Tröltzsch, On the optimal control of the Schlögl-model, Computational Optimization and Applications, 56 (2013), pp. 153-185.

[8] J. Burns AND W.W. Hu, Approximation methods for boundary control of the Boussinesq equations, in 2013 IEEE 52nd Annual Conference on Decision and Control (CDC), IEEE, 2013, pp. 454-459.

[9] E. Casas, C. Ryll, And F. Tröltzsch, Sparse optimal control of the Schlögl and FitzHugh-Nagumo systems, Computational Methods in Applied Mathematics, 13 (2013), pp. 415-442.

[10] K. Chrysafinos, S.P. Filopoulos, and T.K. Papathanasiou, Error estimates for a FitzHugh-Nagumo parameter-dependent reaction-diffusion system, ESAIM: Mathematical Modelling and Numerical Analysis, 47 (2013), pp. 281-304.

[11] E. Fernández-Cara, M. González-Burgos, S. Guerrero, and J.-P. Puel, Null control- 


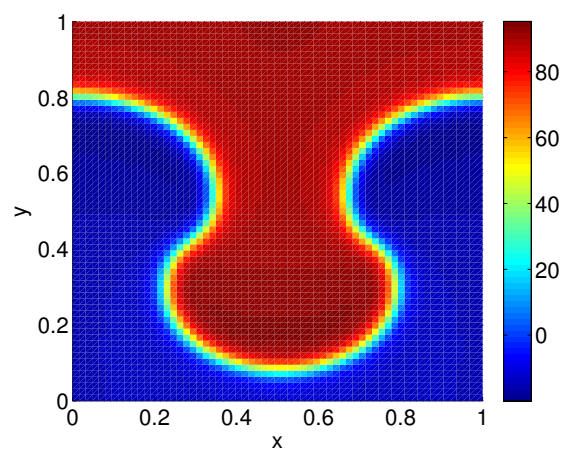

(a) $v(x, y)$ at $t=0$.

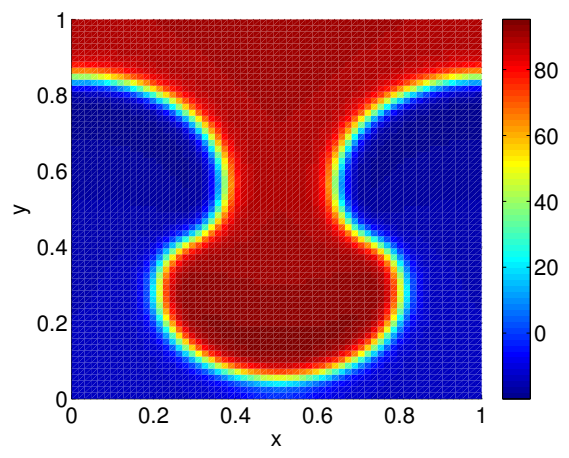

(c) $v(x, y)$ at $t=80$.

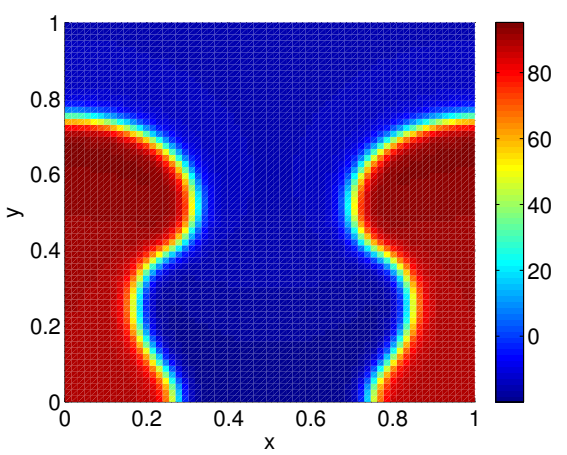

(b) $v(x, y)$ at $t=40$.

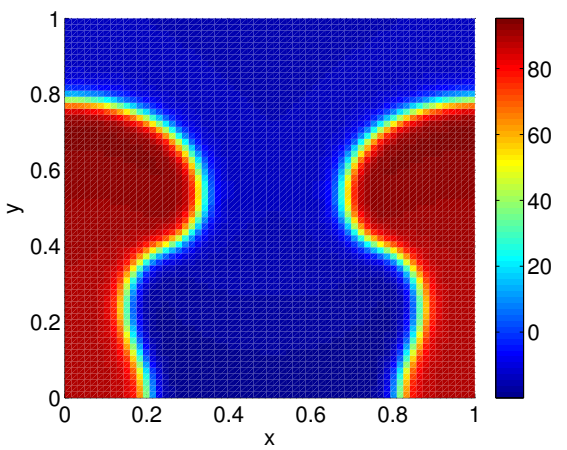

(d) $v(x, y)$ at $t=120$.

Fig. 5.7: Uncontrolled reentry wave.

lability of the heat equation with boundary Fourier conditions: the linear case, ESAIM: Control, Optimisation and Calculus of Variations, 12 (2006), pp. 442-465.

[12] P.C. Franzone, P. Deuflhard, B. Erdmann, J. Lang, and L.F. Pavarino, Adaptivity in space and time for reaction-diffusion systems in electrocardiology, SIAM Journal on Scientific Computing, 28 (2006), pp. 942-962.

[13] P. Grisvard, Commutativité de deux foncteurs d'interpolation et applications, Journal de Mathématiques Pures et Appliquées, 45 (1967), pp. 143-206.

[14] G. GRubb And V.A. Solonnikov, Boundary value problems for the nonstationary NavierStokes equations treated by pseudo-differential methods, Mathematica Scandinavica, 69 (1991), pp. 217-290.

[15] W.W. Hu, Approximation and Control of the Boussinesq Equations with Application to Control of Energy Efficient Building Systems, PhD thesis, Virginia Tech, 2012.

[16] D.E. JACKSON, Existence and regularity for the FitzHugh-Nagumo equations with inhomogeneous boundary conditions, Nonlinear Analysis: Theory, Methods \& Applications, 14 (1990), pp. 201-216.

[17] K. Kunisch, C. NAGAiah, And M. WaGner, A parallel Newton-Krylov method for optimal control of the monodomain model in cardiac electrophysiology, Computing and Visualization in Science, 14 (2011), pp. 257-269.

[18] K. Kunisch And M. Wagner, Optimal control of the bidomain system (I): The monodomain approximation with the Rogers-McCulloch model, Nonlinear Analysis: Real World Applications, 13 (2012), pp. 1525-1550.

[19] K. Kunisch And L. WANG, Time optimal controls of the linear FitzHugh-Nagumo equation 


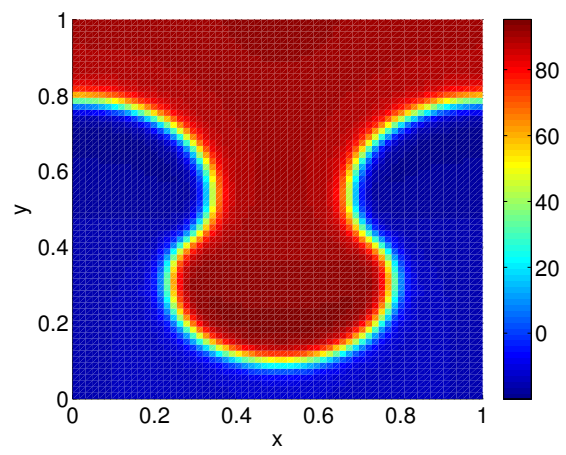

(a) $v(x, y)$ at $t=0$.

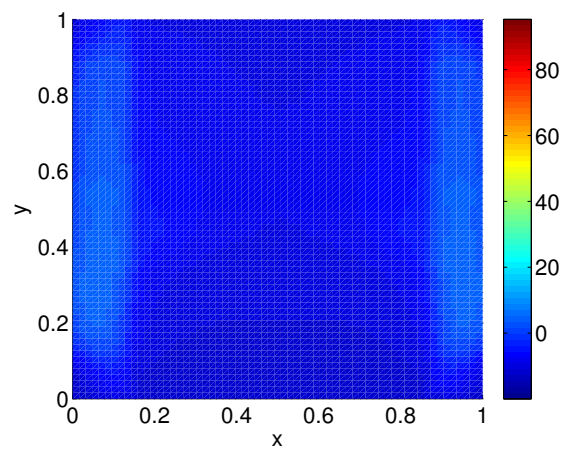

(c) $v(x, y)$ at $t=80$.

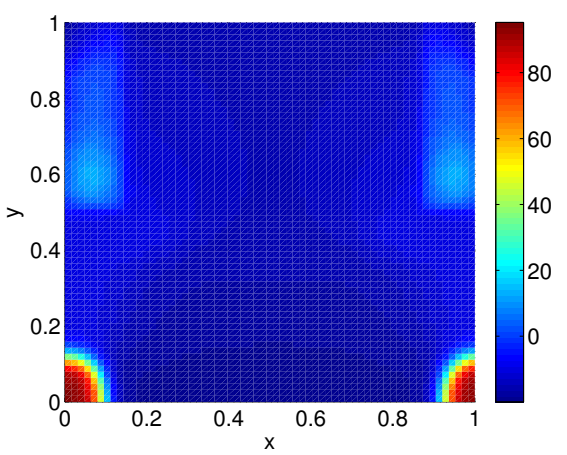

(b) $v(x, y)$ at $t=40$.

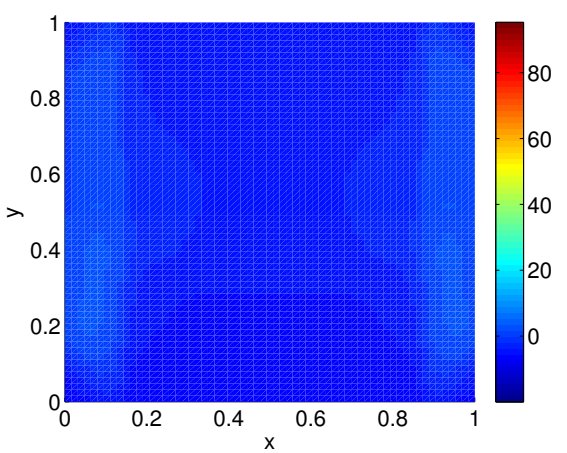

(d) $v(x, y)$ at $t=120$.

Fig. 5.8: Termination of reentry wave.

with pointwise control constraints, Journal of mathematical analysis and applications, 395 (2012), pp. 114-130.

[20] I. LASIECKA, Unified theory for abstract parabolic boundary problems - a semigroup approach, Applied Mathematics and Optimization, 6 (1980), pp. 287-333.

[21] I. Lasiecka and R. Triggiani, Control Theory for Partial Differential Equations: Volume 1, Abstract Parabolic Systems: Continuous and Approximation Theories, vol. 1, Cambridge University Press, 2000.

[22] J.L. Lions and E. Magenes, Non-homogeneous Boundary Value Problems and Applications. Vol. I, Die Grundlehren der mathematischen Wissenschaften in Einzeldarstellungen, Springer-Verlag, Berlin, 1972.

[23] C. Nagaiah, K. Kunisch, and G. Plank, Optimal control approach to termination of re-entry waves in cardiac electrophysiology, Journal of Mathematical Biology, 67 (2013), pp. 359388.

[24] P.A. Nguyen and J.-P. Raymond, Pointwise control of the Boussinesq system, Systems \& Control Letters, 60 (2011), pp. 249-255.

[25] B.F. Nielsen, T.S. Ruud, G.T. Lines, And A. Tveito, Optimal monodomain approximations of the bidomain equations, Applied Mathematics and Computation, 184 (2007), pp. 276290.

[26] A. PAZY, Semigroups of linear operators and applications to partial differential equations, Springer-Verlag, New York, 1983.

[27] A.J. Pritchard And J. ZABCZYK, Stability and stabilizability of infinite dimensional systems, SIAM Review, 23 (1981), pp. 25-52. 
[28] J. Rauch, Global existence for the FitzHugh-Nagumo equations, Communications in Partial Differential Equations, 1 (1976), pp. 609-621.

[29] J. Rauch And J. Smoller, Qualitative theory of the FitzHugh-Nagumo equations, Advances in Mathematics, 27 (1978), pp. 12-44.

[30] J.-P. RAYmond, Feedback boundary stabilization of the two-dimensional Navier-Stokes equations, SIAM Journal on Control and Optimization, 45 (2006), pp. 790-828.

[31] — Feedback boundary stabilization of the three-dimensional incompressible Navier-Stokes equations, Journal de mathématiques pures et appliquées, 87 (2007), pp. 627-669.

[32] L. Thevenet, J.-M. Buchot, And J.-P.Raymond, Nonlinear feedback stabilization of a twodimensional Burgers equation, ESAIM: Control, Optimisation and Calculus of Variations, 16 (2010), pp. 929-955.

[33] L. Tung, A bi-domain model for describing ischemic myocardial DC potentials, PhD thesis, Massachusetts Institute of Technology, 1978.

[34] J. ZABCZYK, Remarks on the algebraic Riccati equation in Hilbert space, Applied Mathematics \& Optimization, 2 (1975), pp. 251-258. 